\title{
Assessment of Minimal Residual Disease by Next Generation Sequencing in Peripheral Blood as a Complementary Tool for Personalized Transplant Monitoring in Myeloid Neoplasms
}

\author{
Paula Aguirre-Ruiz ${ }^{1}$, Beñat Ariceta 1,2, María Cruz Viguria 2,3 (D), María Teresa Zudaire 2,3, \\ Zuriñe Blasco-Iturri ${ }^{1}$, Patricia Arnedo ${ }^{3}$, Almudena Aguilera-Diaz ${ }^{2,4}{ }^{\mathbb{D}}$, Axier Jauregui ${ }^{3}$, \\ Amagoia Mañú 1,2 , Felipe Prosper 2,4,5 (D), María Carmen Mateos 2,3, \\ Marta Fernández-Mercado 1,2,4 ${ }^{D}$, María José Larráyoz ${ }^{1,2}$, Margarita Redondo ${ }^{2,3}$, \\ María José Calasanz ${ }^{1,2}$ (D), Iria Vázquez ${ }^{1,2, *(D)}$ and Eva Bandrés $2,3, *$ (D)
}

1 Hematological Diseases Laboratory, CIMA LAB Diagnostics, University of Navarra, 31008 Pamplona, Navarra, Spain; paguirreruiz@unav.es (P.A.-R.); bariceta@unav.es (B.A.); zurinebi@gmail.com (Z.B.-I.); amanu@unav.es (A.M.); marfermer@yahoo.es (M.F.-M.); mjlarra@unav.es (M.J.L.); mjcal@unav.es (M.J.C.)

2 Navarra Institute for Health Research (IdiSNA), 31008 Pamplona, Navarra, Spain; mc.viguria.alegria@cfnavarra.es (M.C.V.); teresa.zudaire.ripa@navarra.es (M.T.Z.); aadiaz@alumni.unav.es (A.A.-D.); fprosper@unav.es (F.P.); mc.mateos.rodriguez@navarra.es (M.C.M.); am.redondo.izal@navarra.es (M.R.)

3 Hematology Department, Complejo Hospitalario de Navarra, 31008 Pamplona, Navarra, Spain; patrizia.arnedo@gmail.com (P.A.); ajaulop@gmail.com (A.J.)

4 Advanced Genomics Laboratory, Hemato-Oncology, Center for Applied Medical Research (CIMA), 31008 Pamplona, Navarra, Spain

5 Hematology Department, Clinica Universidad de Navarra (CUN), 31008 Pamplona, Navarra, Spain

* Correspondence: ivurio@unav.es (I.V.); eva.bandres.elizalde@navarra.es (E.B.); Tel.: +34-948194700-1000 (I.V.)

Received: 2 November 2020; Accepted: 23 November 2020; Published: 25 November 2020

\begin{abstract}
Patients with myeloid neoplasms who relapsed after allogenic hematopoietic stem cell transplant (HSCT) have poor prognosis. Monitoring of chimerism and specific molecular markers as a surrogate measure of relapse is not always helpful; therefore, improved systems to detect early relapse are needed. We hypothesized that the use of next generation sequencing (NGS) could be a suitable approach for personalized follow-up post-HSCT. To validate our hypothesis, we analyzed by NGS, a retrospective set of peripheral blood (PB) DNA samples previously evaluated by high-sensitive quantitative PCR analysis using insertion/deletion polymorphisms (indel-qPCR) chimerism engraftment. Post-HCST allelic burdens assessed by NGS and chimerism status showed a similar time-course pattern. At time of clinical relapse in 8/12 patients, we detected positive NGS-based minimal residual disease (NGS-MRD). Importantly, in 6/8 patients, we were able to detect NGS-MRD at time points collected prior to clinical relapse. We also confirmed the disappearance of post-HCST allelic burden in non-relapsed patients, indicating true clinical specificity. This study highlights the clinical utility of NGS-based post-HCST monitoring in myeloid neoplasia as a complementary specific analysis to high-sensitive engraftment testing. Overall, NGS-MRD testing in PB is widely applicable for the evaluation of patients following HSCT and highly valuable to personalized early treatment intervention when mixed chimerism is detected.
\end{abstract}

Keywords: next generation sequencing (NGS); chimerism; myeloid leukemia; hematopoietic stem cell transplant (HSCT); minimal residual disease (MRD) 


\section{Introduction}

Allogenic hematopoietic stem cell transplant (HSCT) is a potentially curative treatment in patients with acute myeloid leukemia (AML) and myelodysplastic syndrome (MDS), reducing risk of relapse and improving overall survival [1-3]; however, clinical outcomes still vary among patients [4-7]. Due to the high mortality rate and treatment failures, improved methods of disease status monitoring are clearly needed for patients with myeloid neoplasia following HSCT. Improved surveillance systems may facilitate earlier therapeutic interventions and potentially prevent disease recurrence by tapering immunosuppression, treatment with lymphocyte donor infusion or initiation of anti-neoplastic treatment $[8,9]$. Standard methodologies to detect clinical relapse in myeloid neoplasms currently include: morphologic assessment of the bone marrow (BM), minimal residual disease (MRD) detection by flow cytometry, cytogenetic or molecular genetic marker detection, and hematopoietic chimerism testing. BM histological analysis has a reduced sensitivity for clinical relapse detection [10]. MRD assessment by flow cytometry for AML and MDS is often complicated due to variable sensitivity of patient-specific marker expression profiles, and can also be subject to inter-assay and inter-operator variability [11]. For chimerism analysis, short tandem repeat (STR) polymerase Chain Reaction (PCR) assays are generally applicable to all HSCT patients, but are limited by a sensitivity threshold of $1-5 \%$ [12-14]. Newer techniques to analyze chimerism with higher sensitivity $(0.01-0.1 \%)$ have relatively recently emerged, such as quantitative PCR analysis using insertion/deletion polymorphisms (indel-qPCR) and droplet-digital PCR (ddPCR) [15-17]. However, these assays do not specifically detect the presence of disease, but rather they offer a percentage of recipient's DNA as a surrogate measure for recurrence. This lack of specificity is particularly problematic in chimerism assays, showing high sensitivity, as non-malignant recipient cell lineages may be present in various sample types without representing disease relapse [18]. To maximize sensitivity and specificity, assays such as reverse transcriptase polymerase chain reaction (RT-PCR) may be applied to follow-up specific genetic alterations [19]; however, this is a major limitation in a disease characterized by a striking broad array of different potential oncogenic events across a notable number of genes.

Recently, next generation sequencing (NGS) has been applied to identify clinically relevant variants in AML [20], and persistent allelic burden after chemotherapy has been associated with higher incidence of relapse [21]. Moreover, the presence of genetic variants before HSCT has been associated with higher risk of relapse and shorter overall survival after HSCT [22,23]. Likewise, several studies have demonstrated that the presence of a higher allelic burden at the time of morphologic complete remission is associated with an increased risk of relapse and mortality in AML patients [24,25] and have suggested that the presence of certain genetic variants at morphologic complete remission could be responsible for high risk [26]. Therefore, there has been a great interest to develop high-sensitivity assays to detect any trace of myeloid malignant cells before and after HSCT.

We hypothesized that peripheral blood (PB) serial samples collected for chimerism status monitorization could be useful for NGS analysis, in order to track genetic variants with no additional invasive biopsy procedures. The aim of the present study was to assess the allelic burden in PB using a custom NGS panel alongside measuring the engraftment status using our laboratory's standard-of-care technique for chimerism engraftment monitoring of post-HSCT patients. With these combined datasets, we intended to establish the value of NGS data during chimerism monitorization and assess their combined capacity for personalized early discrimination of molecular relapse, in order to facilitate earlier therapeutic interventions when mixed chimerism (MC) is detected.

\section{Experimental Section}

\subsection{Patient Cohorts and Acquisition of Samples}

A retrospective study, approved by the DIANA project review board (0011-1411-2017-000028), was designed to assess the utility of NGS-MRD detection after HSCT using PB samples collected for routine clinical engraftment analysis. We selected 20 patients (12 AML, $8 \mathrm{MDS} /$ chronic myelomonocytic 
leukemia-CMML) with a variety of chimerism profiles and treatment protocols. Briefly, 12 patients had reduced-intensity conditioning regimen (busulfan plus fludarabine) and 8 patients had a myeloablative conditioning regimen (busulfan plus fludarabine or cyclophosphamide); Graft versus Host Disease (GVHD) prophylaxis was performed with a calcineurin inhibitor (cyclosporine or FK506) with methotrexate; T-depletion was performed for unrelated-donor transplantation; and post-HSCT maintenance therapies were not administered until clinical relapse detection (Table 1). Frequency of chimerism monitoring based on high-risk factors presence and clinical grounds was performed by indel-qPCR analysis on 296 PB DNA samples (mean 15 samples per patient; range 7-29). We selected 75 PB samples for NGS analysis (18 diagnosis, 1 post-induction, and 56 post-HSCT: 45 samples had Mixed chimerism (MC) and 11 had complete chimerism (CC) based on chimerism fluctuations and clinical data (Supplementary Materials Figure S1). Clinical relapse was defined when leukemia blasts were identified by morphological analysis or flow cytometry, or cytogenetic or non-NGS genetic markers were detected. According to these criteria, two groups of patients were studied: patients who relapsed after HSCT $(n=12)$ and patients without relapse at the end of study $(n=8)$. In both groups, we included patients achieving CC at some point during the follow up and patients with MC after HSCT (Supplementary Materials Figure S1). Two donor samples and 8 paired-bone marrow (BM) samples were also included (4 diagnosis, 4 follow-up).

\subsection{Indel-qPCR Chimerism Analysis}

DNA was isolated from $400 \mu \mathrm{L}$ of total PB buffy coat using QIAamp DNA Blood Mini Kit (Qiagen, Hilden, Germany) and quantified by Nanodrop Spectrophotometer (Nanodrop Technologies, Wilmington, NC, USA). Baseline donor and recipient DNA were genotyped with the KMR Genotyping Kit (GenDx, Utrecht, The Netherlands) and informative markers were selected (positive in recipient and negative in donor). Chimerism presence was tested by KMR Track Kit (GeneDx), with post-HSCT DNA (150 ng) and pre-HSCT recipient DNA (10 ng), and the chimerism percentages, represented as host-DNA percentages, were determined using the ddCt method according to the manufacturer's instructions [27]. We defined complete chimerism (CC) as host-DNA percentage inferior to $0.01 \%$ and mixed chimerism (MC) as host-DNA percentage above this threshold.

\subsection{Next Generation Sequencing (NGS)}

DNA samples were quantified using Qubit dsDNA BR Assay Kit on a Qubit 3.0 Fluorometer (Life Technologies, Carlsbad, CA, USA), and quality was assessed by DNA genomic kit on a Tape Station 4100 (Agilent Technologies, Santa Clara, CA, USA). Samples at diagnosis and post-HSCT were analyzed with a custom pan-myeloid panel targeting 48 myeloid genes described by Aguilera-Diaz et al. [28]. Libraries were carried out following manufacturer's instructions, quantified using the Qubit dsDNA HS Assay Kit on a Qubit 3.0 Fluorometer (Life Technologies), and quality was assessed using the D1000 Kit on the 4100 Tape Station (Agilent Technologies); 8 pooled libraries were normalized at $4 \mathrm{nM}$ and pair-end sequenced on a MiSeq Sequencer (Illumina, San Diego, CA, USA) with $251 \times 2$ cycles using the Reagent Kit V3 600 cycles cartridge (Illumina, San Diego, CA, USA). 
Table 1. Clinical and therapeutic characteristics of myeloid patients included in this study. Genetic risk was defined by specific scores: ELN for AML, IPPS-R for MDS and CPSS for CMML; pre-transplant disease status was determined by analysis of bone marrow morphology; MRD pre-transplant was determined by flow-cytometry or the presence of a single-molecular marker; and HSCT conditioning regime was selected accordingly to patient fitness.

\begin{tabular}{|c|c|c|c|c|c|c|c|c|c|c|c|c|c|c|c|c|}
\hline UPN & Sex & $\begin{array}{l}\text { Age at } \\
\text { HSCT }\end{array}$ & Diagnosis & $\begin{array}{l}\text { AML/MDS } \\
\text { Diagnosis }\end{array}$ & $\begin{array}{l}\text { Genetic } \\
\text { Risk }\end{array}$ & $\begin{array}{l}\text { Classical Genetic } \\
\text { Markers }\end{array}$ & $\begin{array}{l}\text { NGS Genetic Markers } \\
\text { Pre-HSCT }\end{array}$ & $\begin{array}{c}\text { Pre-HSCT } \\
\text { Disease } \\
\text { Status }\end{array}$ & $\begin{array}{l}\text { MRD } \\
\text { Pre-HSCT } \\
\text { Status }\end{array}$ & $\begin{array}{l}\text { Days from } \\
\text { Diagnosis } \\
\text { to HSCT }\end{array}$ & $\begin{array}{c}\text { HSCT } \\
\text { Conditioning } \\
\text { Regimen }\end{array}$ & $\begin{array}{l}\text { Immunosupression } \\
\text { Treatment }\end{array}$ & $\begin{array}{l}\text { HLA Antigen } \\
\text { Match }\end{array}$ & $\begin{array}{l}\text { Chimerism } \\
\text { Profile after } \\
\text { HSCT }\end{array}$ & $\begin{array}{l}\text { Chimerism } \\
\text { Profile at } \\
\text { Relapse }\end{array}$ & $\begin{array}{l}\text { Clinical } \\
\text { Outcome }\end{array}$ \\
\hline 1 & M & 18 & JMML & de novo & intermediate & $46, \mathrm{XY}(30)$ & NRAS-p.Gln61Lys & CR1 & positive & 137 & MA (BuCy) & $\begin{array}{l}\text { FK506 + MTX + } \\
\text { ATG }\end{array}$ & $\begin{array}{l}\text { fully matched } \\
\text { unrelated donor }\end{array}$ & $\mathrm{CC}$ & MC & relapse \\
\hline 2 & $\mathrm{~F}$ & 66 & AML & de novo & intermediate & $\begin{array}{c}46, \mathrm{XX}, \mathrm{t} \\
(4 ; 12)(\mathrm{q} 12 ; \mathrm{p} 13)(14) \\
\text { /46,XX(16) } \\
\text { FLT3-ITD(-) }\end{array}$ & $\begin{array}{c}\text { IDH2-p.Arg172Lys } \\
\text { NF1-p.Ile1603Val } \\
\text { DNMT3A-p.Val895Met } \\
\text { DNMT3A-p.Arg729Gln }\end{array}$ & CR1 & positive & 188 & $\begin{array}{l}\text { RIC (FLU + } \\
\text { BU2) }\end{array}$ & FK506 + MTX & $\begin{array}{l}\text { fully matched } \\
\text { unrelated donor }\end{array}$ & CC & MC & relapse \\
\hline 3 & $\mathrm{~F}$ & 70 & AML & Secondary & adverse & $\begin{array}{c}46, \mathrm{XX}, \mathrm{del}(5 \mathrm{~g})(22 / 25) \\
/ 46, \mathrm{XX}(3 / 25)\end{array}$ & $\begin{array}{l}\text { TP53-p.Arg273Cys } \\
\text { NRAS-p.Gly13Asp } \\
\text { SH2B3-p.? }\end{array}$ & Not CR & positive & 231 & $\begin{array}{c}\text { RIC (FLU + } \\
\text { BU2) }\end{array}$ & FK506 + MTX & $\begin{array}{l}\text { fully matched } \\
\text { sibling donor }\end{array}$ & $\mathrm{CC}$ & MC & relapse \\
\hline 5 & $\mathrm{~F}$ & 65 & AML & de novo & adverse & $\begin{array}{l}\text { hypodiploid complex } \\
\text { karyotype }\end{array}$ & $\begin{array}{c}\text { TP53-p.Val173Met } \\
\text { GATA2-p.Gly149Arg }\end{array}$ & CR1 & positive & 121 & $\begin{array}{c}\text { RIC (FLU + } \\
\text { BU2) }\end{array}$ & $\mathrm{CS}+\mathrm{MTX}$ & $\begin{array}{l}\text { fully matched } \\
\text { sibling donor }\end{array}$ & CC & MC & relapse \\
\hline 4 & $\mathrm{~F}$ & 61 & AML & Secondary & adverse & $\begin{array}{c}47, \mathrm{XX},-3, \\
\operatorname{del}(5)(\mathrm{q} 1 \mathrm{q} 333), \\
+8,-17,+21,+21(6) \\
/ 48, \text { idem, }+20(3) \\
/ 46, \mathrm{XX}(7)\end{array}$ & $\begin{array}{c}\text { TP53-p.? } \\
\text { ETV6-p.Arg291Glyfs*25 }\end{array}$ & CR1 & positive & 144 & $\begin{array}{c}\text { RIC (FLU + } \\
\text { BU2) }\end{array}$ & $\begin{array}{l}\text { FK506 + MTX + } \\
\text { ATG }\end{array}$ & $\begin{array}{l}\text { single antigen } \\
\text { mismatch } \\
\text { unrelated donor }\end{array}$ & MC & MC & relapse \\
\hline 11 & M & 37 & AML & de novo & intermediate & $46, X Y(25)$ & $\begin{array}{l}\text { PTPN11-p.Gly503Glu } \\
\text { RUNX1-p.? }\end{array}$ & CR1 & ND & 129 & $\mathrm{MA}(\mathrm{BuCy})$ & $\begin{array}{l}\text { CS + MTX + } \\
\text { CAMPATH }\end{array}$ & $\begin{array}{l}\text { fully matched } \\
\text { unrelated donor }\end{array}$ & MC & $\mathrm{MC}$ & relapse \\
\hline 12 & M & 69 & MDS & Secondary & adverse & $\begin{array}{c}\text { trisomy } 8 \text { and } \\
\text { monosomy } 7\end{array}$ & $\begin{array}{c}\text { DNMT3A-p.Arg326Cys } \\
\text { U2AF1-p.Ser34Phe }\end{array}$ & CR1 & ND & 177 & $\begin{array}{c}\text { RIC (FLU + } \\
\text { BU2) }\end{array}$ & $\begin{array}{l}\text { CS + MTX + } \\
\text { CAMPATH }\end{array}$ & $\begin{array}{c}\text { fully matched } \\
\text { unrelated donor }\end{array}$ & MC & MC & relapse \\
\hline 13 & F & 57 & MDS & de novo & adverse & $\begin{array}{c}45, X X,-7(4) \\
/ 45, X,-X(3) / 46, X X(13) \\
\end{array}$ & KRAS-p.Gly12Cys & Not CR & positive & 259 & $\begin{array}{c}\text { MA (FLU + } \\
\text { BU4) }\end{array}$ & FK506 + MTX & $\begin{array}{l}\text { fully matched } \\
\text { unrelated donor }\end{array}$ & MC & MC & relapse \\
\hline 14 & $\mathrm{~F}$ & 59 & AML & de novo & intermediate & $\begin{array}{l}47, \mathrm{XX},+4(5 / 20) \\
/ 46, \mathrm{XX}(15 / 20)\end{array}$ & $\begin{array}{c}\text { FLT3-p.Val592Ala } \\
\text { NPM1-p.Trp288Cysfs*12 } \\
\text { DNMT3A-p.Arg882His } \\
\text { KRAS-p.Gly12Asp } \\
\text { KMT2A-p.Gln147Arg }\end{array}$ & CR1 & negative & 161 & $\begin{array}{c}\text { RIC (FLU + } \\
\text { BU2) }\end{array}$ & FK506 + MTX & $\begin{array}{l}\text { fully matched } \\
\text { sibling donor }\end{array}$ & MC & MC & relapse \\
\hline 18 & F & 56 & MDS & Secondary & adverse & $\begin{array}{l}46, X X, \operatorname{inv}(3) \\
(\mathrm{q} 21 \mathrm{q} 26)(20)\end{array}$ & $\begin{array}{l}\text { PHF6-p.Arg274Ter } \\
\text { SF3B1-p.Ala708Pro } \\
\text { CUX1-p.Arg554Gln }\end{array}$ & CR1 & positive & 155 & $\begin{array}{c}\text { RIC (FLU + } \\
\text { BU2) }\end{array}$ & FK506 + MTX & $\begin{array}{l}\text { fully matched } \\
\text { sibling donor }\end{array}$ & MC & $\mathrm{MC}$ & relapse \\
\hline 19 & M & 59 & CMML & de novo & intermediate & $45, \mathrm{X}, \mathrm{Y}(1) / 46, \mathrm{XY}(3)$ & 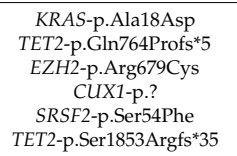 & Not CR & ND & 1750 & $\begin{array}{c}\text { RIC (FLU + } \\
\text { BU2) }\end{array}$ & FK506 + MTX & $\begin{array}{l}\text { fully matched } \\
\text { sibling donor }\end{array}$ & MC & $\mathrm{MC}$ & relapse \\
\hline 20 & F & 62 & MDS & de novo & adverse & $\begin{array}{c}47, X X,+8(17 / 20) \\
/ 46, X X(3 / 20)\end{array}$ & ND & CR1 & negative & 239 & $\begin{array}{c}\text { RIC (FLU + } \\
\text { BU2) }\end{array}$ & FK506 + MTX & $\begin{array}{l}\text { fully matched } \\
\text { sibling donor }\end{array}$ & MC & MC & relapse \\
\hline 6 & F & 45 & AML & de novo & adverse & $\begin{array}{c}46, X X(13) \\
\text { FLT3-ITD }(+)\end{array}$ & $\begin{array}{l}\text { FLT3-ITD-p.Tyr597 } \\
\text {-Glu611dup } \\
\text { NPM1-p.Trp288Cysfs"12 } \\
\text { DNMT3A-p.Leu } \\
\text { 639Serfs"12 }\end{array}$ & CR1 & positive & 138 & MA (BuCy) & $\begin{array}{l}\text { FK506 + MTX + } \\
\text { ATG }\end{array}$ & $\begin{array}{l}\text { fully matched } \\
\text { unrelated donor }\end{array}$ & $\mathrm{CC}$ & - & remission \\
\hline
\end{tabular}


Table 1. Cont.

\begin{tabular}{|c|c|c|c|c|c|c|c|c|c|c|c|c|c|c|c|c|}
\hline UPN & Sex & $\begin{array}{l}\text { Age at } \\
\text { HSCT }\end{array}$ & Diagnosis & $\begin{array}{l}\text { AML/MDS } \\
\text { Diagnosis }\end{array}$ & $\begin{array}{l}\text { Genetic } \\
\text { Risk }\end{array}$ & $\begin{array}{l}\text { Classical Genetic } \\
\text { Markers }\end{array}$ & $\begin{array}{l}\text { NGS Genetic Markers } \\
\text { Pre-HSCT }\end{array}$ & $\begin{array}{c}\text { Pre-HSCT } \\
\text { Disease } \\
\text { Status }\end{array}$ & $\begin{array}{l}\text { MRD } \\
\text { Pre-HSCT } \\
\text { Status }\end{array}$ & $\begin{array}{l}\text { Days from } \\
\text { Diagnosis } \\
\text { to HSCT }\end{array}$ & $\begin{array}{c}\text { HSCT } \\
\text { Conditioning } \\
\text { Regimen }\end{array}$ & $\begin{array}{c}\text { Immunosupression } \\
\text { Treatment }\end{array}$ & $\begin{array}{l}\text { HLA Antigen } \\
\text { Match }\end{array}$ & $\begin{array}{l}\text { Chimerism } \\
\text { Profile after } \\
\text { HSCT }\end{array}$ & $\begin{array}{l}\text { Chimerism } \\
\text { Profile at } \\
\text { Relapse }\end{array}$ & $\begin{array}{l}\text { Clinical } \\
\text { Outcome }\end{array}$ \\
\hline 7 & $\mathrm{~F}$ & 42 & AML & de novo & intermediate & $\begin{array}{c}46, \mathrm{XX}(24 / 25) \\
/ 47, \mathrm{XX},+8(1 / 25]) \\
\text { nuc } \\
\text { ish(D8Z }(\mathrm{D} 2 \times 3)(87 / 145)\end{array}$ & $\begin{array}{c}\text { IDH2-p.parg172Lys } \\
\text { SH2B3-p.Ser213Arg } \\
\text { RUNX1-p.Ser390Profs*? }\end{array}$ & CR1 & positive & 136 & MA (BuCy) & FK506 + MTX & $\begin{array}{l}\text { fully matched } \\
\text { sibling donor }\end{array}$ & $\mathrm{CC}$ & - & remission \\
\hline 10 & M & 39 & AML & de novo & adverse & $\begin{array}{c}46, \mathrm{XY}, \mathrm{t}(3 ; 3)(\mathrm{q} 21 ; \mathrm{q} 26) \\
\text { FLT3-ITD }(+)\end{array}$ & $\begin{array}{c}\text { FLT3-ITD-p.Asp586 } \\
\text {-Glu598dup } \\
\text { NPM1-p.Trp288Cysfs*12 } \\
\text { CUX1-p.Arg219GIn } \\
\text { GATA2-p.Gly135Trpfs*50 }\end{array}$ & CR1 & negative & 170 & $\begin{array}{c}\mathrm{MA} \text { (FLU + } \\
\text { BU4) }\end{array}$ & FK506 + MTX & $\begin{array}{l}\text { fully matched } \\
\text { sibling donor }\end{array}$ & $\mathrm{CC}$ & - & remission \\
\hline 15 & $\mathrm{~F}$ & 61 & AML & Secondary & adverse & $\begin{array}{l}45, X X,-7(6 / 20) \\
/ 46, X X(14 / 20)\end{array}$ & $\begin{array}{l}\text { DNMT3A-p.Arg8824His } \\
\text { IDH1-p.Arg132Cys } \\
\text { DNMT3A-p.Phe868Ser }\end{array}$ & CR1 & ND & 159 & $\begin{array}{c}\text { RIC (FLU + } \\
\text { BU2) }\end{array}$ & FK506 + MTX & $\begin{array}{l}\text { fully matched } \\
\text { sibling donor }\end{array}$ & $\mathrm{CC}$ & - & remission \\
\hline 16 & M & 39 & MDS & de novo & adverse & 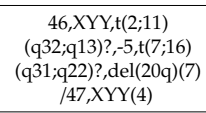 & $\begin{array}{l}\text { TP53-p.Arg267Trp } \\
\text { RUNX1-p.Arg139Gln } \\
\text { SRSF2-p.Pro95Leu } \\
\text { NF1-p.Leu380Phe }\end{array}$ & Not CR & positive & 262 & $\begin{array}{l}\text { MA (FLU + } \\
\text { BU4) }\end{array}$ & FK506 + MTX & $\begin{array}{l}\text { fully matched } \\
\text { sibling donor }\end{array}$ & $\mathrm{CC}$ & - & remission \\
\hline 17 & M & 41 & MDS & de novo & adverse & $\begin{array}{c}46, X Y, \operatorname{del}(12 p)(7) \\
/ 46, X Y(18)\end{array}$ & $\begin{array}{l}\text { U2AF1-p.Ser34Phe } \\
\text { CALR-p.Glu380Gly }\end{array}$ & Not CR & positive & 88 & MA (BuCy) & $\mathrm{CS}+\mathrm{MTX}$ & $\begin{array}{l}\text { fully matched } \\
\text { sibling donor }\end{array}$ & $\mathrm{CC}$ & - & remission \\
\hline 8 & $\mathrm{~F}$ & 56 & AML & de novo & adverse & $\begin{array}{c}47, \mathrm{XX},+8, \mathrm{t}(5 ; ; ; ; 11 ; 13) \\
(\mathrm{q} 33 ; \mathrm{p} 22 ; \mathrm{q} 23 ; \mathrm{q} 13)\end{array}$ & $\begin{array}{l}\text { KRAS-p.Gly13Asp } \\
\text { PTPN11-p.Ala72Thr }\end{array}$ & CR1 & negative & 161 & $\underset{\text { BU2) }}{\mathrm{RIC} \text { (FLU + }}$ & $\begin{array}{l}\text { CS + MTX + } \\
\text { CAMPATH }\end{array}$ & $\begin{array}{l}\text { single antigen } \\
\text { mismatch } \\
\text { unrelated donor }\end{array}$ & MC & - & remission \\
\hline 9 & M & 68 & AML & de novo & intermediate & $46, X Y(20)$ & $\begin{array}{l}\text { ASXL1-p.Gly646Trpfs*12 } \\
\text { SRSF2-p.Pro95is } \\
\text { KMT2A-p.Leu999Phe } \\
\text { NF1-p.Leu2714Val } \\
\text { RUNX1-p.Asn82Asp }\end{array}$ & CR1 & ND & 162 & $\begin{array}{c}\text { RIC (FLU + } \\
\text { BU2) }\end{array}$ & $\begin{array}{l}\text { CS + MTX + } \\
\text { CAMPATH }\end{array}$ & $\begin{array}{l}\text { fully matched } \\
\text { unrelated donor }\end{array}$ & MC & - & remission \\
\hline
\end{tabular}

$\mathrm{UPN}=$ unique patient number; $\mathrm{M}$ = male; $\mathrm{F}$ = female; $\mathrm{AML}$ = acute myeloid leukemia; $\mathrm{MDS}$ = myelodysplastic syndrome; JMML = juvenile myelomonocytic leukemia; $\mathrm{CMML}=$ chronic myelomonocytic leukemia; MRD = minimal residual disease; ELN = European LeukemiaNet; IPPS-R = Revised International Prognostic Scoring System; CPSS = CMML-specific prognostic scoring system; ND = not determined; $\mathrm{CR}$, complete response; $\mathrm{HSCT}=$ hematopoietic stem cell transplant; MA = myeloablative; RIC = reduced intensity conditioning; $\mathrm{BuCy}$ = busulfan-cyclophosphamide; FLU = fludarabine; BU2 = busulfan 2 days; BU4 = busulfan 4 days; FK506 = tacrolimus; MTX = methotrexate; ATG = antithymocyte globulin; $\mathrm{CS}=$ cyclosporin $\mathrm{A} ; \mathrm{CC}=$ complete chimerism; $\mathrm{MC}=$ mixed chimerism . 


\subsection{Variant Data Analysis}

Fastq files were uploaded onto SOPHiA DDM software (SOPHiA GENETICS, Saint Sulpice, Switzerland) for alignment, variant calling, and annotation, filtering out intronic and intergenic variants. Aligned reads were manually curated with the Integrative Genomics Viewer (IGV) software (Broad Institute, Cambridge, MA, USA).

In addition, two in-house hotspot variant calling analyses were performed using VarScan version 2.4.2 [29] and GATK version 4.0.8.1 Mutect2 [30] to detect variants with variant allele frequency (VAF) below $1 \%$ threshold. The filtering values for VarScan analysis were: strand bias; minimum coverage: 2 ; minimum supporting reads at a position to call variants: 2; minimum base quality at a position to count a read: 1 ; and minimum VAF: $10-5$. For Mutect 2 analysis, the parameters were: minimum base quality required to consider a base for calling was reduced to 1 , the minimum phred-scaled confidence threshold at which variants should be called to 1 and the maximum number of reads to retain per alignment start position was disabled. Mutect2 was run in tumor-only mode and with hotspots as interval list to reduce computing time. Variants from both methods were manually curated to confirm the hotspots selected for each patient.

Clinical classification of the resulting variants was individually reviewed according to the Spanish Group of Myelodysplastic Syndromes guidelines [31]. Post-HSCT monitoring was performed considering all NGS-trackable variants, meaning variants that: (i) were classified as pathogenic, likely pathogenic, or variants of uncertain significance (VUS); (ii) had a minimum coverage of 500 reads; (iii) had a minimum of 12 reads of the alternative allele; and (iv) had a VAF $\geq 0.1 \%$ with at least one of their time points with VAF $>5 \%$. Regarding MRD by NGS in post-HSCT, a sample was considered NGS-MRD positive when a variant with clinical relevance, including pathogenic and/or likely pathogenic variants, was detected.

\section{Results}

\subsection{Assessment of the NGS Sensitivity on PB Samples}

First, we assessed the sensitivity of NGS on PB samples in comparison to BM paired samples by Pearson correlation test. We compared $4 \mathrm{~PB}$ and BM samples at diagnosis, and similar VAF were detected showing similar sensitivity $\left(R^{2}=0.9891 ; p\right.$-value $\left.<0.0001\right)$. Besides, comparison of $4 \mathrm{~PB}$ and BM samples at follow-up times showed high correlation $\left(R^{2}=0.9978 ; p\right.$-value $\left.<0.0001\right)$ (Supplementary Materials Figure S2).

These results showed similar sensitivity of NGS on PB and BM samples both for the diagnosis and follow-up, confirming that PB samples are also suitable for molecular testing when BM is not available.

\subsection{Identification of NGS Variants in PB of Myeloid Neoplasms}

We analyzed samples collected at the time of diagnosis $(n=18)$ or at post-induction treatment time $(n=1)$; no sample before HSCT was available for unique patient number (UPN)20. The remaining 19 patients showed a total of 57 variants. Considering variants of UPN20 and de novo acquired variants during the follow-up, the number of total detected variants increased to 63 (mean 3.15 per patient). These variants classified as pathogenic $(n=31)$, likely pathogenic $(n=3)$, and VUS $(n=29)$ showed a broad range of VAF $(0.21-88.84 \%)$ and were spread across 25 genes. NGS data help to better stratify 3 AML patients shifting from intermediate to high risk group due to the presence of RUNX1 variants (UPN7, UPN9, UPN11) (Table 2). 
Table 2. NGS variants identified in the 20 patients during the disease time course. Information of the variants detected with the pan-myeloid panel includes VAF percentage and sequencing depth for all time points. For variants with VAF below 1\% results from VarScan (SNV) and Mutect2 (indels) in-house analysis are plotted.

\begin{tabular}{|c|c|c|c|c|c|c|c|c|c|c|c|c|c|c|c|c|}
\hline UPN & Gene & $\mathrm{Chr}$ & Position & Consequence & c.DNA & Protein & Classification & Diagnosis & Post-TM & $\begin{array}{c}\text { Post-HSCT } \\
1 \\
\end{array}$ & $\begin{array}{c}\text { Post-HSCT } \\
2\end{array}$ & $\begin{array}{c}\text { Post-HSCT } \\
3 \\
\end{array}$ & $\begin{array}{c}\text { Post-HSCT } \\
4 \\
\end{array}$ & $\begin{array}{c}\text { Post-HSCT } \\
5 \\
\end{array}$ & Relapse & Post-Relapse \\
\hline \multirow{3}{*}{1} & NRAS & 1 & 115256530 & missense & c. $181 \mathrm{C}>\mathrm{A}$ & p.Gln61Lys & Pathogenic & $\begin{array}{l}45.38 \% \\
8951 x\end{array}$ & & ND & ND & ND & $\begin{array}{c}12 \% \\
6457 x \\
\end{array}$ & \multirow{3}{*}{ - } & $\begin{array}{l}14.07 \% \\
5872 x\end{array}$ & \\
\hline & WT1 & 11 & 32417914 & frameshift & c.1086dupA & p.Arg363Thrfs*5 & $\begin{array}{c}\text { Uncertain } \\
\text { significance }\end{array}$ & ND & & ND & ND & ND & $\begin{array}{c}10 \% \\
7688 \mathrm{x} \\
\end{array}$ & & $\begin{array}{c}13 \% \\
7444 x \\
\end{array}$ & - \\
\hline & WT1 & 11 & 32417910 & frameshift & $\begin{array}{l}\text { c.1077_1090dup } \\
\text { GACTCTTGTA } \\
\text { CGGT }\end{array}$ & p.Ser364Ter & $\begin{array}{l}\text { Uncertain } \\
\text { significance }\end{array}$ & ND & & ND & ND & $\begin{array}{l}0.21 \% \\
6200 x\end{array}$ & $\begin{array}{c}9 \% \\
7694 x\end{array}$ & & $\begin{array}{c}13 \% \\
7400 x\end{array}$ & \\
\hline \multirow{4}{*}{2} & IDH2 & 15 & 90631838 & missense & c. $515 \mathrm{G}>\mathrm{A}$ & p.Arg172Lys & Pathogenic & $\begin{array}{l}13.91 \% \\
4667 \mathrm{x} \\
\end{array}$ & \multirow{4}{*}{-} & ND & ND & \multirow{4}{*}{-} & \multirow{4}{*}{ - } & \multirow{4}{*}{ - } & $\begin{array}{l}0.40 \% \\
3716 \mathrm{x} \\
\end{array}$ & $\begin{array}{l}1.62 \% \\
5002 x \\
\end{array}$ \\
\hline & NF1 & 17 & 29652872 & missense & c. $4807 \mathrm{~A}>\mathrm{G}$ & p.lle1603Val & $\begin{array}{c}\text { Uncertain } \\
\text { significance }\end{array}$ & $\begin{array}{l}48.96 \% \\
3619 \mathrm{x} \\
\end{array}$ & & ND & ND & & & & $\begin{array}{l}0.42 \% \\
3352 x \\
\end{array}$ & $\begin{array}{l}1.60 \% \\
4634 \mathrm{x} \\
\end{array}$ \\
\hline & DNMT3A & 2 & 25457204 & missense & c. $2683 \mathrm{G}>\mathrm{A}$ & p.Val895Met & $\begin{array}{c}\text { Uncertain } \\
\text { significance }\end{array}$ & $\begin{array}{l}12.61 \% \\
5688 \mathrm{x} \\
\end{array}$ & & ND & ND & & & & $\begin{array}{l}0.47 \% \\
4510 \mathrm{x} \\
\end{array}$ & $\begin{array}{l}1.81 \% \\
5967 x \\
\end{array}$ \\
\hline & DNMT3A & 2 & 25463307 & missense & $c .2186 \mathrm{G}>\mathrm{A}$ & p.Arg729Gln & $\begin{array}{c}\text { Uncertain } \\
\text { significance }\end{array}$ & $\begin{array}{l}12.04 \% \\
6036 \mathrm{x} \\
\end{array}$ & & ND & ND & & & & $\begin{array}{l}0.37 \% \\
4884 \mathrm{x} \\
\end{array}$ & $\begin{array}{l}1.41 \% \\
6183 x \\
\end{array}$ \\
\hline \multirow{3}{*}{3} & TP53 & 17 & 7577121 & missense & c. $817 \mathrm{C}>\mathrm{T}$ & p.Arg273Cys & Pathogenic & $\begin{array}{l}19.97 \% \\
3445 \mathrm{x}\end{array}$ & \multirow{3}{*}{ - } & ND & ND & $\begin{array}{l}0.58 \% \\
5165 x\end{array}$ & \multirow{3}{*}{ - } & \multirow{3}{*}{ - } & $\begin{array}{l}4.03 \% \\
6688 \mathrm{x}\end{array}$ & \\
\hline & NRAS & 1 & 115258744 & missense & $c .38 \mathrm{G}>\mathrm{A}$ & p.Gly13Asp & Pathogenic & $\begin{array}{l}4.20 \% \\
4020 \mathrm{x} \\
\end{array}$ & & ND & ND & ND & & & ND & - \\
\hline & SH2B3 & 12 & 111885351 & splice site & c. $1236+3 \mathrm{~A}>\mathrm{G}$ & p.? & $\begin{array}{c}\text { Uncertain } \\
\text { significance }\end{array}$ & $\begin{array}{l}2.97 \% \\
3810 \mathrm{x} \\
\end{array}$ & & $\begin{array}{l}.99 \% \\
3810 \mathrm{x} \\
\end{array}$ & $\begin{array}{l}1.43 \% \\
4186 \mathrm{x} \\
\end{array}$ & $\begin{array}{l}1.48 \% \\
4987 x \\
\end{array}$ & & & $\begin{array}{l}3.67 \% \\
4792 x \\
\end{array}$ & \\
\hline \multirow{2}{*}{5} & TP53 & 17 & 7578413 & missense & c. $517 \mathrm{G}>\mathrm{A}$ & p.Val173Met & Pathogenic & $\begin{array}{l}1.32 \% \\
7719 x\end{array}$ & \multirow[b]{2}{*}{-} & $\begin{array}{l}0.32 \% \\
4999 \mathrm{x}\end{array}$ & ND & \multirow[b]{2}{*}{-} & \multirow[b]{2}{*}{ - } & \multirow[b]{2}{*}{-} & ND & \multirow[b]{2}{*}{-} \\
\hline & GATA2 & 3 & 128204996 & missense & c. $445 \mathrm{G}>\mathrm{A}$ & p.Gly149Arg & $\begin{array}{c}\text { Uncertain } \\
\text { significance }\end{array}$ & $\begin{array}{l}51.46 \% \\
6528 \mathrm{x} \\
\end{array}$ & & $\begin{array}{l}4.32 \% \\
6246 \mathrm{x} \\
\end{array}$ & ND & & & & $\begin{array}{l}0.68 \% \\
3691 x \\
\end{array}$ & \\
\hline \multirow[b]{2}{*}{4} & TP53 & 17 & 7578370 & splice site & c. $559+1 \mathrm{G}>\mathrm{A}$ & p? & Pathogenic & $\begin{array}{l}28.94 \% \\
7888 x \\
\end{array}$ & & ND & ND & \multirow[b]{2}{*}{ - } & \multirow{2}{*}{ - } & \multirow[b]{2}{*}{-} & ND & ND \\
\hline & ETV6 & 12 & 12022762 & frameshift & c.870delC & p.Arg291Glyfs"25 & $\begin{array}{c}\text { Uncertain } \\
\text { significance }\end{array}$ & $\begin{array}{l}17.69 \% \\
8934 x \\
\end{array}$ & & ND & ND & & & & ND & ND \\
\hline \multirow[b]{2}{*}{11} & PTPN11 & 12 & 112926888 & missense & c. $1508 \mathrm{G}>\mathrm{A}$ & p.Gly503Glu & Pathogenic & $\begin{array}{l}32.91 \% \\
5585 \mathrm{x} \\
\end{array}$ & & ND & & \multirow{2}{*}{ - } & \multirow{2}{*}{ - } & \multirow[b]{2}{*}{-} & ND & \\
\hline & RUNX1 & 21 & 36252852 & splice site & c. $427+2 \mathrm{~T}>\mathrm{C}$ & p? & $\begin{array}{c}\text { Uncertain } \\
\text { significance }\end{array}$ & $\begin{array}{l}35.04 \% \\
1096 \mathrm{x} \\
\end{array}$ & & ND & - & & & & ND & - \\
\hline \multirow[b]{2}{*}{12} & DNMT3A & 2 & 25470498 & missense & $\mathrm{c} .976 \mathrm{C}>\mathrm{T}$ & p.Arg326Cys & $\begin{array}{c}\text { Likely } \\
\text { pathogenic }\end{array}$ & $\begin{array}{l}7.12 \% \\
5648 \mathrm{x} \\
\end{array}$ & & $\begin{array}{l}0.79 \% \\
2341 \mathrm{x} \\
\end{array}$ & $\begin{array}{l}0.30 \% \\
7718 x \\
\end{array}$ & \multirow[b]{2}{*}{ - } & & & $\begin{array}{l}0.48 \% \\
2935 x \\
\end{array}$ & \\
\hline & U2AF1 & 21 & 44524456 & missense & c. $101 \mathrm{C}>\mathrm{T}$ & p.Ser34Phe & Pathogenic & $\begin{array}{l}5.35 \% \\
5363 x\end{array}$ & & ND & ND & & - & $\begin{array}{l}- \\
-\end{array}$ & ND & - \\
\hline 13 & KRAS & 12 & 25398285 & missense & c. $34 \mathrm{G}>\mathrm{T}$ & p.Gly12Cys & Pathogenic & $\begin{array}{l}7.54 \% \\
2919 x\end{array}$ & - & ND & $\begin{array}{l}0.58 \% \\
1733 x\end{array}$ & - & - & - & $\begin{array}{l}2.38 \% \\
3237 x\end{array}$ & - \\
\hline
\end{tabular}


Table 2. Cont

\begin{tabular}{|c|c|c|c|c|c|c|c|c|c|c|c|c|c|c|c|c|}
\hline UPN & Gene & Chr & Position & Consequence & c.DNA & Protein & Classification & Diagnosis & Post-TM & $\begin{array}{c}\text { Post-HSCT } \\
1 \\
\end{array}$ & $\begin{array}{c}\text { Post-HSCT } \\
2 \\
\end{array}$ & $\begin{array}{c}\text { Post-HSCT } \\
3 \\
\end{array}$ & $\begin{array}{c}\text { Post-HSCT } \\
4 \\
\end{array}$ & $\begin{array}{c}\text { Post-HSCT } \\
5 \\
\end{array}$ & Relapse & Post-Relapse \\
\hline \multirow{5}{*}{14} & FLT3 & 13 & 28608281 & missense & c. $1775 \mathrm{~T}>\mathrm{C}$ & p.Val592Ala & Pathogenic & $\begin{array}{l}23.42 \% \\
3151 x \\
\end{array}$ & \multirow{5}{*}{ - } & \multirow{5}{*}{ - } & \multirow{5}{*}{ - } & \multirow{5}{*}{ - } & \multirow{5}{*}{ - } & \multirow{5}{*}{ - } & ND & ND \\
\hline & $\begin{array}{l}\text { NPM1 } \\
\text { Type A }\end{array}$ & 5 & 170837543 & frameshift & c.860_863dupTCTG & p.Trp288Cysfs"12 & Pathogenic & $\begin{array}{l}15.27 \% \\
1821 x \\
\end{array}$ & & & & & & & ND & ND \\
\hline & DNMT3A & 2 & 25457242 & missense & c. $2645 \mathrm{G}>\mathrm{A}$ & p.Arg882His & Pathogenic & $\begin{array}{l}36.24 \% \\
3797 x \\
\end{array}$ & & & & & & & $\begin{array}{l}2.22 \% \\
2832 x\end{array}$ & $\begin{array}{r}2.07 \% \\
13045 x \\
\end{array}$ \\
\hline & KRAS & 12 & 25398284 & missense & $c .35 \mathrm{G}>\mathrm{A}$ & p.Gly12Asp & Pathogenic & $\begin{array}{l}1.94 \% \\
2167 \mathrm{x} \\
\end{array}$ & & & & & & & ND & ND \\
\hline & KMT2A & 11 & 118339497 & missense & c. $440 \mathrm{~A}>\mathrm{G}$ & p.Gln147Arg & $\begin{array}{c}\text { Uncertain } \\
\text { significance }\end{array}$ & $\begin{array}{l}28.84 \% \\
2691 x \\
\end{array}$ & & & & & & & ND & ND \\
\hline \multirow{3}{*}{18} & PHFG & $\mathrm{x}$ & 133549136 & stop codon & c. $820 \mathrm{C}>\mathrm{T}$ & p.Arg274Ter & $\begin{array}{c}\text { Likely } \\
\text { pathogenic }\end{array}$ & $\begin{array}{l}12.74 \% \\
2834 x\end{array}$ & \multirow{3}{*}{ - } & ND & \multirow{3}{*}{ - } & \multirow{3}{*}{ - } & \multirow{3}{*}{ - } & \multirow{3}{*}{ - } & ND & \multirow{3}{*}{ - } \\
\hline & SF3B1 & 2 & 198266810 & missense & c. $2122 \mathrm{G}>\mathrm{C}$ & p.Ala708Pro & $\begin{array}{c}\text { Uncertain } \\
\text { significance }\end{array}$ & $\begin{array}{l}17.97 \% \\
3016 \mathrm{x} \\
\end{array}$ & & ND & & & & & ND & \\
\hline & CUX1 & 7 & 101923357 & missense & c. $1661 \mathrm{G}>\mathrm{A}$ & p.Arg554Gln & $\begin{array}{c}\text { Uncertain } \\
\text { significance }\end{array}$ & $\begin{array}{l}49.19 \% \\
3015 x\end{array}$ & & $\begin{array}{l}3.27 \% \\
6597 x\end{array}$ & & & & & $\begin{array}{l}3.31 \% \\
2446 x\end{array}$ & \\
\hline \multirow{6}{*}{19} & KRAS & 12 & 25398266 & missense & c. $53 \mathrm{C}>\mathrm{A}$ & p.Ala18Asp & Pathogenic & $\begin{array}{l}41.46 \% \\
2383 x\end{array}$ & \multirow{6}{*}{ - } & $\begin{array}{l}1.69 \% \\
5756 x\end{array}$ & \multirow{6}{*}{ - } & \multirow{6}{*}{ - } & \multirow{6}{*}{ - } & \multirow{6}{*}{ - } & $\begin{array}{l}10.82 \% \\
1303 \mathrm{x}\end{array}$ & \\
\hline & TET2 & 4 & 106157384 & frameshift & c.2290dupC & p.Gln764Profs*5 & Pathogenic & $\begin{array}{l}39.64 \% \\
3042 x \\
\end{array}$ & & $\begin{array}{l}1.71 \% \\
8269 \mathrm{x} \\
\end{array}$ & & & & & $\begin{array}{l}16.83 \% \\
2400 x \\
\end{array}$ & \\
\hline & EZH2 & 7 & 148506462 & missense & c. $2035 \mathrm{C}>\mathrm{T}$ & p.Arg679Cys & $\begin{array}{c}\text { Likely } \\
\text { pathogenic }\end{array}$ & $\begin{array}{l}84.49 \% \\
2243 x \\
\end{array}$ & & $\begin{array}{l}3.28 \% \\
6309 \mathrm{x} \\
\end{array}$ & & & & & $\begin{array}{l}34.12 \% \\
1603 x \\
\end{array}$ & - \\
\hline & CuX1 & 7 & 101713618 & splice site & c. $223-1 \mathrm{G}>\mathrm{T}$ & $\mathrm{p} ?$ ? & $\begin{array}{c}\text { Uncertain } \\
\text { significance }\end{array}$ & $\begin{array}{l}88.84 \% \\
1945 \mathrm{x} \\
\end{array}$ & & $\begin{array}{l}2.36 \% \\
4997 x \\
\end{array}$ & & & & & $\begin{array}{l}39.76 \% \\
1484 \mathrm{x} \\
\end{array}$ & \\
\hline & SRSF 2 & 17 & 74733082 & missense & c. $161 \mathrm{C}>\mathrm{T}$ & p.Ser54Phe & $\begin{array}{l}\text { Uncertain } \\
\text { significance }\end{array}$ & $\begin{array}{l}43.34 \% \\
2469 \mathrm{x}\end{array}$ & & $\begin{array}{r}1.65 \% \\
10315 x \\
\end{array}$ & & & & & $\begin{array}{l}18.52 \% \\
2921 x \\
\end{array}$ & \\
\hline & TET2 & 4 & 106197221 & frameshift & c.5557_5558dup & p.Ser1853Argfs"35 & $\begin{array}{c}\text { Uncertain } \\
\text { significance }\end{array}$ & $\begin{array}{l}41.81 \% \\
3449 \mathrm{x} \\
\end{array}$ & & $\begin{array}{l}1.49 \% \\
9252 x \\
\end{array}$ & & & & & $\begin{array}{l}19.31 \% \\
3729 \mathrm{x} \\
\end{array}$ & \\
\hline \multirow{4}{*}{20} & SRSF2 & 17 & 74732959 & missense & $c .284 \mathrm{C}>\mathrm{G}$ & p.Pro95Arg & Pathogenic & \multirow{4}{*}{ - } & \multirow{4}{*}{ - } & $\begin{array}{r}9.07 \% \\
11465 \mathrm{x} \\
\end{array}$ & \multirow{4}{*}{ - } & \multirow{4}{*}{-} & \multirow{4}{*}{ - } & \multirow{4}{*}{ - } & $\begin{array}{l}42.72 \% \\
11317 \mathrm{x} \\
\end{array}$ & \\
\hline & CUX1 & 7 & 101848405 & missense & c. $3118 \mathrm{G}>\mathrm{A}$ & p.Val1040Met & $\begin{array}{c}\text { Uncertain } \\
\text { significance }\end{array}$ & & & $\begin{array}{l}15.12 \% \\
4187 \mathrm{x} \\
\end{array}$ & & & & & $\begin{array}{l}42.32 \% \\
3852 x \\
\end{array}$ & \\
\hline & TET2 & 4 & 106190851 & missense & c. $4129 \mathrm{~T}>\mathrm{G}$ & p.Phe1377Val & $\begin{array}{c}\text { Uncertain } \\
\text { significance }\end{array}$ & & & $\begin{array}{l}10.52 \% \\
6340 \mathrm{x} \\
\end{array}$ & & & & & $\begin{array}{l}74.32 \% \\
5947 \mathrm{x} \\
\end{array}$ & \\
\hline & RUNX1 & 21 & 36259163 & missense & c. $247 \mathrm{~A}>\mathrm{C}$ & p.Lys83Gln & $\begin{array}{c}\text { Uncertain } \\
\text { significance }\end{array}$ & & & $\begin{array}{l}1.41 \% \\
3757 x \\
\end{array}$ & & & & & $\begin{array}{l}5.83 \% \\
4271 \mathrm{x} \\
\end{array}$ & \\
\hline \multirow{3}{*}{6} & FLT3-ITD & 13 & 28608223 & inframe & c.1788_1832dup & p.Tyr597_Glu611dup & Pathogenic & $\begin{array}{l}51 \% \\
6880 \mathrm{x} \\
\end{array}$ & \multirow{3}{*}{ - } & ND & ND & ND & \multirow{3}{*}{ - } & & & \\
\hline & $\begin{array}{l}\text { NPM1 } \\
\text { Type A }\end{array}$ & 5 & 170837543 & frameshift & c.860_863dupTCTG & p.Trp288Cysfs"12 & Pathogenic & $\begin{array}{l}36.09 \% \\
3497 x \\
\end{array}$ & & ND & ND & ND & & - & - & - \\
\hline & DNMT3A & 2 & 25466788 & frameshift & c.1914delT & p.Leu639Serfs"12 & $\begin{array}{c}\text { Uncertain } \\
\text { significance }\end{array}$ & $\begin{array}{l}43.80 \% \\
7175 x\end{array}$ & & ND & ND & ND & & & & \\
\hline
\end{tabular}


Table 2. Cont.

\begin{tabular}{|c|c|c|c|c|c|c|c|c|c|c|c|c|c|c|c|c|}
\hline UPN & Gene & $\mathrm{Chr}$ & Position & Consequence & c.DNA & Protein & Classification & Diagnosis & Post-TM & $\begin{array}{c}\text { Post-HSCT } \\
1 \\
\end{array}$ & $\begin{array}{c}\text { Post-HSCT } \\
2\end{array}$ & $\begin{array}{c}\text { Post-HSCT } \\
3 \\
\end{array}$ & $\begin{array}{c}\text { Post-HSCT } \\
4 \\
\end{array}$ & $\begin{array}{c}\text { Post-HSCT } \\
5 \\
\end{array}$ & Relapse & Post-Relapse \\
\hline \multirow{3}{*}{7} & IDH2 & 15 & 90631838 & missense & c. $515 \mathrm{G}>\mathrm{A}$ & p.Arg172Lys & Pathogenic & $\begin{array}{l}16.09 \% \\
6232 x \\
\end{array}$ & \multirow{3}{*}{ - } & ND & ND & \multirow{3}{*}{ - } & \multirow{3}{*}{ - } & \multirow{3}{*}{ - } & \multirow{3}{*}{ - } & \multirow{3}{*}{ - } \\
\hline & SH2B3 & 12 & 111856588 & missense & c. $639 \mathrm{C}>\mathrm{A}$ & p.Ser213Arg & $\begin{array}{c}\text { Uncertain } \\
\text { significance }\end{array}$ & $\begin{array}{l}47.90 \% \\
5635 \mathrm{x} \\
\end{array}$ & & $\begin{array}{l}0.79 \% \\
2404 x \\
\end{array}$ & ND & & & & & \\
\hline & RUNX1 & 21 & 36164626 & frameshift & c.1167delC & p.Ser390Profs*? & $\begin{array}{c}\text { Uncertain } \\
\text { significance }\end{array}$ & $\begin{array}{l}15.24 \% \\
4613 x \\
\end{array}$ & & ND & ND & & & & & \\
\hline \multirow{4}{*}{10} & FLT3-ITD & 13 & 28608261 & inframe & c.1756_1794dup39 & p.Asp586_Glu598dup & Pathogenic & $\begin{array}{c}43 \% \\
4503 x \\
\end{array}$ & \multirow{4}{*}{ - } & ND & ND & \multirow{4}{*}{ - } & \multirow{4}{*}{ - } & \multirow{4}{*}{ - } & \multirow{4}{*}{ - } & \multirow{4}{*}{ - } \\
\hline & $\begin{array}{l}\text { NPM1 } \\
\text { Type D }\end{array}$ & 5 & 170837544 & frameshift & c.863_864i-CCTG & p.Trp288Cysfs"12 & Pathogenic & $\begin{array}{l}36.74 \% \\
2730 x \\
\end{array}$ & & ND & ND & & & & & \\
\hline & CUX1 & 7 & 101758502 & missense & c. $656 \mathrm{G}>\mathrm{A}$ & p.Arg219Gln & $\begin{array}{c}\text { Uncertain } \\
\text { significance }\end{array}$ & $\begin{array}{l}47.41 \% \\
3634 \mathrm{x} \\
\end{array}$ & & $\begin{array}{l}1.19 \% \\
2010 x \\
\end{array}$ & ND & & & & & \\
\hline & GATA2 & 3 & 128205042 & frameshift & c.399_430 & p.Gly135Trpfs" 50 & $\begin{array}{c}\text { Uncertain } \\
\text { significance }\end{array}$ & $\begin{array}{l}45.04 \% \\
4043 \mathrm{x} \\
\end{array}$ & & ND & ND & & & & & \\
\hline \multirow{3}{*}{15} & DNMT3A & 2 & 25457242 & missense & c. $2645 \mathrm{G}>\mathrm{A}$ & p.Arg882His & Pathogenic & $\begin{array}{l}10.33 \% \\
6246 \mathrm{x} \\
\end{array}$ & \multirow{3}{*}{ - } & ND & & \multirow{3}{*}{ - } & \multirow{3}{*}{ - } & \multirow{3}{*}{ - } & \multirow{3}{*}{ - } & \multirow{3}{*}{ - } \\
\hline & IDH1 & 2 & 209113113 & missense & $c .394 \mathrm{C}>\mathrm{T}$ & p.Arg132Cys & Pathogenic & $\begin{array}{l}3.82 \% \\
5495 \mathrm{x}\end{array}$ & & ND & - & & & & & \\
\hline & DNMT3A & 2 & 25457284 & missense & c. $2603 \mathrm{~T}>\mathrm{C}$ & p.Phe868Ser & $\begin{array}{l}\text { Uncertain } \\
\text { significance }\end{array}$ & $\begin{array}{l}5.53 \% \\
6092 x \\
\end{array}$ & & ND & & & & & & \\
\hline \multirow{4}{*}{16} & TP53 & 17 & 7577139 & missense & c.799C $>\mathrm{T}$ & p.Arg267Trp & Pathogenic & $\begin{array}{l}51.86 \% \\
3922 \mathrm{x} \\
\end{array}$ & \multirow{4}{*}{-} & $\begin{array}{l}1.72 \% \\
7751 \mathrm{x} \\
\end{array}$ & ND & \multirow{4}{*}{ - } & \multirow{4}{*}{ - } & \multirow{4}{*}{ - } & \multirow{4}{*}{ - } & \multirow{4}{*}{ - } \\
\hline & RUNX1 & 21 & 36252865 & missense & c. $416 \mathrm{G}>\mathrm{A}$ & p.Arg139Gln & Pathogenic & $\begin{array}{l}12.11 \% \\
1024 \mathrm{x} \\
\end{array}$ & & ND & ND & & & & & \\
\hline & SRSF2 & 17 & 74732959 & missense & $c .284 \mathrm{C}>\mathrm{T}$ & p.Pro95Leu & Pathogenic & $\begin{array}{l}5.40 \% \\
3539 \mathrm{x} \\
\end{array}$ & & ND & ND & & & & & \\
\hline & NF1 & 17 & 29528130 & missense & c. $1138 \mathrm{C}>\mathrm{T}$ & p.Leu380Phe & $\begin{array}{c}\text { Uncertain } \\
\text { significance }\end{array}$ & $\begin{array}{l}35.46 \% \\
2033 x \\
\end{array}$ & & $\begin{array}{l}44 \% \\
3011 x \\
\end{array}$ & $\begin{array}{r}51 \% \\
1413 x \\
\end{array}$ & & & & & \\
\hline \multirow[b]{2}{*}{17} & U2AF1 & 21 & 44524456 & missense & $c .101 \mathrm{C}>\mathrm{T}$ & p.Ser34Phe & Pathogenic & $\begin{array}{l}25.20 \% \\
3012 x \\
\end{array}$ & & ND & ND & & & & & \\
\hline & CALR & 19 & 13054612 & missense & c.1139A > G & p.Glu380Gly & $\begin{array}{l}\text { Uncertain } \\
\text { significance }\end{array}$ & $\begin{array}{l}51.90 \% \\
3703 x \\
\end{array}$ & - & $\begin{array}{l}1.22 \% \\
3865 \mathrm{x}\end{array}$ & ND & - & - & - & - & - \\
\hline & KRAS & 12 & 25398281 & missense & $c .38 \mathrm{G}>\mathrm{A}$ & p.Gly13Asp & Pathogenic & $\begin{array}{l}38.32 \% \\
5128 x \\
\end{array}$ & & ND & ND & ND & & & & \\
\hline 8 & PTPN11 & 12 & 112888198 & missense & $c .214 \mathrm{G}>\mathrm{A}$ & p.Ala72Thr & Pathogenic & $\begin{array}{l}4.63 \% \\
6042 x \\
\end{array}$ & & ND & ND & ND & - & - & & \\
\hline & ASXL1 & 20 & 31022441 & frameshift & c.1934dupG & p.Gly646Trpfs"12 & Pathogenic & & $\begin{array}{l}1.40 \% \\
6069 \mathrm{x} \\
\end{array}$ & $\begin{array}{l}1.49 \% \\
3293 x \\
\end{array}$ & $\begin{array}{l}1.61 \% \\
2231 x \\
\end{array}$ & $\begin{array}{l}1.62 \% \\
3769 \mathrm{x} \\
\end{array}$ & $\begin{array}{l}7.18 \% \\
5675 \mathrm{x} \\
\end{array}$ & $\begin{array}{c}16 \% \\
14672 x \\
\end{array}$ & & \\
\hline & SRSF2 & 17 & 74732959 & missense & c. $284 \mathrm{C}>\mathrm{A}$ & p.Pro95His & Pathogenic & & $1.21 \% 6677 x$ & ND & ND & $1.12 \% 3479 x$ & $7.11 \% 4879 x$ & $16.52 \% 15740 \mathrm{x}$ & & \\
\hline 9 & KMT2A & 11 & 118344839 & missense & c. $2965 \mathrm{C}>\mathrm{T}$ & p.Leu989Phe & $\begin{array}{c}\text { Uncertain } \\
\text { significance }\end{array}$ & - & $48.66 \% 6178 x$ & $\mathrm{x} \quad \mathrm{ND}$ & $0.69 \% 2036 x$ & $1.61 \% 5476 x$ & $6.87 \% 4539 x$ & $12 \% 4007 x$ & - & - \\
\hline & $N F 1$ & 17 & 29687547 & missense & c. $8140 \mathrm{C}>\mathrm{G}$ & p.Leu2714Val & $\begin{array}{c}\text { Uncertain } \\
\text { significance }\end{array}$ & & $49.82 \% 5221 \mathrm{x}$ & $\begin{array}{ll}x & N D\end{array}$ & $\mathrm{ND}$ & $1.87 \% 4547 x$ & $5.71 \% 4117 x$ & $8.50 \% 3624 x$ & & \\
\hline & RUNX1 & 21 & 36259166 & missense & c. $244 \mathrm{~A}>\mathrm{G}$ & p.Asn82Asp & $\begin{array}{c}\text { Uncertain } \\
\text { significance }\end{array}$ & & $0.97 \% 3005 x$ & ND & ND & $0.69 \% 2188 x$ & $6.27 \% 3143 x$ & $11.85 \% 5427 x$ & & \\
\hline
\end{tabular}

$\mathrm{UPN}=$ unique patient number; $\mathrm{Chr}=$ chromosome; TM = treatment; HSCT = hematopoietic stem cell transplantation; ND = not detected; hyphen $(-)=\mathrm{NGS}$ analysis not performed. 
To determine the value of molecular NGS-MRD for the discrimination of relapse or non-relapse when MC was detected, only variants classified as pathogenic and likely pathogenic $(n=34)$ were considered (Table 3). The patient without sample before HSCT (UPN20) with a NGS-MRD variant during the follow-up was also included for molecular relapse associated analysis. The NGS-MRD variants were spread across 16 genes (KRAS, TP53, DNMT3A, FLT3, NPM1, SRSF2, IDH2, NRAS, PTPN11, ASXL1, EZH2, IDH1, PHF6, RUNX1, TET2, U2AF1), and included 27 single-nucleotide variants(SNV) and 7 indels. The most frequent altered genes were KRAS and TP53 (4 patients), DNMT3A, FLT3, NPM1, and SRSF2 (3 patients) (Table 2).

Table 3. Correlation between chimerism and presence of molecular variants for the 20 HSCT patients. Results show the percentage of chimerism in total peripheral blood and the presence of molecular markers detected by NGS for all time points during the disease course.

\begin{tabular}{|c|c|c|c|c|c|c|c|}
\hline UPN & Diagnosis & Patient Group & Moment of Sample & $\begin{array}{l}\text { Days after } \\
\text { HSCT }\end{array}$ & $\%$ Chimerism & $\begin{array}{l}\text { NGS-Trackable } \\
\text { Variants }^{1}\end{array}$ & $\begin{array}{l}\text { NGS-MRD } \\
\text { Variants }{ }^{2}\end{array}$ \\
\hline \multirow{6}{*}{1} & \multirow{6}{*}{ JMML } & \multirow{6}{*}{ Relapse } & Before HSCT & - & - & Positive & Positive \\
\hline & & & Post-HSCT & 100 & $0.95 \%$ & Negative & Negative \\
\hline & & & Post-HSCT & 600 & $<0.01 \%$ & Negative & Negative \\
\hline & & & Post-HSCT & 850 & $0.3 \%$ & Positive & Negative \\
\hline & & & Post-HSCT & 950 & $12 \%$ & Positive & Positive \\
\hline & & & Relapse & 985 & $64 \%$ & Positive & Positive \\
\hline \multirow{5}{*}{2} & \multirow{5}{*}{ AML } & \multirow{5}{*}{ Relapse } & Before HSCT & - & - & Positive & Positive \\
\hline & & & Post-HSCT & 250 & $<0.01 \%$ & Negative & Negative \\
\hline & & & Post-HSCT & 360 & $0.09 \%$ & Negative & Negative \\
\hline & & & Relapse & 380 & $0.67 \%$ & Positive & Positive \\
\hline & & & Post-Relapse & 400 & $2.24 \%$ & Positive & Positive \\
\hline \multirow{5}{*}{3} & \multirow{5}{*}{ AML } & \multirow{5}{*}{ Relapse } & Before HSCT & - & - & Positive & Positive \\
\hline & & & Post-HSCT & 90 & $6.87 \%$ & Positive & Negative \\
\hline & & & Post-HSCT & 580 & $<0.01 \%$ & Positive & Negative \\
\hline & & & Post-HSCT & 650 & $0.12 \%$ & Positive & Positive \\
\hline & & & Relapse & 690 & $7.7 \%$ & Positive & Positive \\
\hline \multirow{4}{*}{5} & \multirow{4}{*}{ AML } & \multirow{4}{*}{ Relapse } & Before HSCT & - & - & Positive & Positive \\
\hline & & & Post-HSCT & 90 & $6.8 \%$ & Positive & Positive \\
\hline & & & Post-HSCT & 540 & $<0.01 \%$ & Negative & Negative \\
\hline & & & Relapse & 1350 & $1.41 \%$ & Positive & Negative \\
\hline \multirow{5}{*}{4} & \multirow{5}{*}{ AML } & \multirow{5}{*}{ Relapse } & Before HSCT & - & - & Positive & Positive \\
\hline & & & Post-HSCT & 100 & $0.1 \%$ & Negative & Negative \\
\hline & & & Post-HSCT & 300 & $0.12 \%$ & Negative & Negative \\
\hline & & & Relapse & 410 & $0.2 \%$ & Negative & Negative \\
\hline & & & Post-Relapse & 470 & $0.34 \%$ & Negative & Negative \\
\hline \multirow{3}{*}{11} & \multirow{3}{*}{ AML } & \multirow{3}{*}{ Relapse } & Before HSCT & - & - & Positive & Positive \\
\hline & & & Post-HSCT & 100 & $19 \%$ & Negative & Negative \\
\hline & & & Relapse & 130 & $67 \%$ & Negative & Negative \\
\hline \multirow{4}{*}{12} & \multirow{4}{*}{ MDS } & \multirow{4}{*}{ Relapse } & Before HSCT & - & - & Positive & Positive \\
\hline & & & Post-HSCT & 600 & $3.3 \%$ & Positive & Positive \\
\hline & & & Post-HSCT & 720 & $2.7 \%$ & Positive & Positive \\
\hline & & & Relapse & 820 & $2.85 \%$ & Positive & Positive \\
\hline \multirow{4}{*}{13} & \multirow{4}{*}{ MDS } & \multirow{4}{*}{ Relapse } & Before HSCT & - & - & Positive & Positive \\
\hline & & & Post-HSCT & 45 & $3.6 \%$ & Negative & Negative \\
\hline & & & Post-HSCT & 80 & $5.2 \%$ & Positive & Positive \\
\hline & & & Relapse & 100 & $11.6 \%$ & Positive & Positive \\
\hline
\end{tabular}


Table 3. Cont.

\begin{tabular}{|c|c|c|c|c|c|c|c|}
\hline UPN & Diagnosis & Patient Group & Moment of Sample & $\begin{array}{l}\text { Days after } \\
\text { HSCT }\end{array}$ & $\%$ Chimerism & $\begin{array}{c}\text { NGS-Trackable } \\
\text { Variants }^{1}\end{array}$ & $\begin{array}{l}\text { NGS-MRD } \\
\text { Variants }^{2}\end{array}$ \\
\hline \multirow{3}{*}{14} & \multirow{3}{*}{ AML } & \multirow{3}{*}{ Relapse } & Before HSCT & - & - & Positive & Positive \\
\hline & & & Relapse & 60 & $5.5 \%$ & Positive & Positive \\
\hline & & & Post-Relapse & 140 & $<0.01 \%$ & Positive & Positive \\
\hline \multirow{3}{*}{18} & \multirow{3}{*}{ MDS } & \multirow{3}{*}{ Relapse } & Before HSCT & - & - & Positive & Positive \\
\hline & & & Post-HSCT & 90 & $6.2 \%$ & Positive & Negative \\
\hline & & & Relapse & 180 & $5.5 \%$ & Positive & Negative \\
\hline \multirow{3}{*}{19} & \multirow{3}{*}{ MDS } & \multirow{3}{*}{ Relapse } & Before HSCT & - & - & Positive & Positive \\
\hline & & & Post-HSCT & 80 & $6.7 \%$ & Positive & Positive \\
\hline & & & Relapse & 120 & $19 \%$ & Positive & Positive \\
\hline \multirow{3}{*}{20} & \multirow{3}{*}{ MDS } & \multirow{3}{*}{ Relapse } & Before HSCT & - & - & NA & NA \\
\hline & & & Post-HSCT & 90 & $29 \%$ & Positive & Positive \\
\hline & & & Relapse & 130 & $100 \%$ & Positive & Positive \\
\hline \multirow{4}{*}{6} & \multirow{4}{*}{ AML } & \multirow{4}{*}{ Remission } & Before HSCT & - & - & Positive & Positive \\
\hline & & & Post-HSCT & 90 & $0.02 \%$ & Negative & Negative \\
\hline & & & Post-HSCT & 300 & $0.01 \%$ & Negative & Negative \\
\hline & & & Post-HSCT & 820 & $<0.01 \%$ & Negative & Negative \\
\hline \multirow{3}{*}{7} & \multirow{3}{*}{ AML } & \multirow{3}{*}{ Remission } & Before HSCT & - & - & Positive & Positive \\
\hline & & & Post-HSCT & 90 & $1.02 \%$ & Positive & Negative \\
\hline & & & Post-HSCT & 420 & $<0.01 \%$ & Negative & Negative \\
\hline \multirow{3}{*}{10} & \multirow{3}{*}{ AML } & \multirow{3}{*}{ Remission } & Before HSCT & - & - & Positive & Positive \\
\hline & & & Post-HSCT & 110 & $1.79 \%$ & Positive & Negative \\
\hline & & & Post-HSCT & 170 & $<0.01 \%$ & Negative & Negative \\
\hline \multirow{2}{*}{15} & \multirow{2}{*}{ AML } & \multirow{2}{*}{ Remission } & Before HSCT & - & - & Positive & Positive \\
\hline & & & Post-HSCT & 60 & $0.85 \%$ & Negative & Negative \\
\hline \multirow{3}{*}{16} & & & Before HSCT & - & - & Positive & Positive \\
\hline & MDS & Remission & Post-HSCT & 90 & $3.85 \%$ & Positive & Positive \\
\hline & & & Post-HSCT & 360 & $<0.01 \%$ & Negative & Negative \\
\hline & & & Before HSCT & - & - & Positive & Positive \\
\hline 17 & MDS & Remission & Post-HSCT & 30 & $1.6 \%$ & Positive & Negative \\
\hline & & & Post-HSCT & 160 & $<0.01 \%$ & Negative & Negative \\
\hline & & & Before HSCT & - & - & Positive & Positive \\
\hline 8 & $\mathrm{AMT}$ & Romicion & Post-HSCT & 90 & $15.4 \%$ & Negative & Negative \\
\hline 0 & RIVIL & 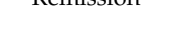 & Post-HSCT & 200 & $14.9 \%$ & Negative & Negative \\
\hline & & & Post-HSCT & 1140 & $33 \%$ & Negative & Negative \\
\hline & & & Before HSCT & - & - & Positive & Positive \\
\hline & & & Post-HSCT & 100 & $0.21 \%$ & Positive & Positive \\
\hline 9 & $\mathrm{AMI}$ & Remission & Post-HSCT & 370 & $1.7 \%$ & Positive & Positive \\
\hline & & & Post-HSCT & 1250 & $2 \%$ & Positive & Positive \\
\hline & & & Post-HSCT & 1360 & $10 \%$ & Positive & Positive \\
\hline & & & Post-HSCT & 1550 & $26 \%$ & Positive & Positive \\
\hline
\end{tabular}

${ }^{1}$ NGS-trackable variants: including variants classified as pathogenic, likely pathogenic, or VUS. ${ }^{2}$ NGS-MRD variants: including variants classified as pathogenic or likely pathogenic. UPN = unique patient number; HSCT = hematopoietic stem cell transplant; NGS = next generation sequencing; VUS= variant of unknown significance; $\mathrm{MRD}=$ minimal residual disease; $\mathrm{NA}=$ not available; Hyphen= not performed (Chimerism assay is done after HSCT).

We found that 14 patients had 25 variants in clonal hematopoiesis of indeterminate potential (CHIP)-associated genes (DNMT3A, SRSF2, CUX1, TET2, TP53, UA2F1, ASXL1, SF3B1) [32,33]. Of those, 8 patients harbored more than 1 variant ( 6 patients with 2 variants, 1 patient with 3 variants, and 1 patient with 4 variants).

These results demonstrate that NGS performed on PB samples is also suitable to characterize the molecular clonal heterogeneity of the myeloid malignancies, and provides useful information to improve the risk stratification of myeloid patients. 


\subsection{Molecular Variants and Chimerism Dynamics after Allogenic HSCT}

Low level of host-DNA can be detected in PB for several months after transplant by high-sensitive indel-qPCR assay. Therefore, to determine the presence of molecular markers in the same PB samples would be useful for the interpretation of these low levels of MC. In our study, kinetics of chimerism and genetic variants detected in 56 samples post-HSCT showed a similar time-course pattern (Table 3). Accordingly to chimerism status, 45 samples had MC and 11 had CC. Specifically, in 31/45 (69\%) of the samples with MC, we detected NGS-variants; even with MC values below $5 \%$ ( 15 samples). We did not detect any variants in $14 / 45$ of the samples with MC; 8 of those had MC values below $1 \%$. In addition, within the 11/56 samples with CC, 9 samples ( $82 \%$ ) showed no molecular variants (Table 3 ).

These results indicate that NGS might provide additional useful information to chimerism status data during follow-up after-HSCT.

\subsection{NGS-MRD Specificity in PB Samples from Non-Relapsed Patients}

In order to establish the specificity of molecular NGS-MRD in PB, we monitored the pathogenic or likely pathogenic variants of 8 patients in remission with different chimerism status for at least 12 months after HSCT (20 samples).

According to chimerism profile, in 6/8 patients, MC decreased until CC was reached (Figure 1), with a mean time of 220 days (range 90-360 days) (Table 3). During MC time, no NGS-MRD variants were detected in $5 / 6$ patients and in $3 / 6$ patients only VUS was present (Table 3 ). For UPN16, a pathogenic variant detected during MC time disappeared when CC status was achieved, while a VUS in the NF1 gene (VAF $\approx 50 \%$ ) confirmed in his sibling-donor was detected at all follow-up samples (Table 2, Supplementary Materials Figure S3).

In two non-relapsed patients, we detected an increase of MC after HSCT. In UPN8, although MC was persistent and high (>10\% host-DNA), no variant was detected at days 90,180 , and 1135 post-HSCT. Surprisingly, for patient UPN9, despite the fact that relapse had never occurred, we detected an increase of VAF for the variants in the CHIP-associated genes ASXL1 and SRSF2 concomitant to the MC increase (Table 2, Supplementary Materials Figure S4). In summary, NGS-MRD was negative at the last time point tested in 7/8 non-relapsed patients, and in 5 of those, the NGS-MRD status totally correlated with CC.

\subsection{NGS-MRD Sensitivity in PB Samples from Relapsed Patients}

To assess the sensitivity of NGS-MRD detection in PB samples during post-HSCT follow-up, we tested 36 samples from the relapsed group (12 patients). In 8 patients $(67 \%)$, positive NGS-MRD correlated with the presence of MC at the time of clinical relapse (Table 3). All variants detected at relapse were already present at diagnosis; and additionally, in UPN1, two new acquired VUS, not present in his HSC donor, were also identified, suggesting clonal evolution and disease progression (Figure 2). In two patients with CC and negative NGS-MRD (UPN2, UPN3), NGS-MRD was detected when slight increase in chimerism was measured $(0.67 \%$ and $0.12 \%$ host-DNA)(Figure 2 and Supplementary Materials Figure S5). Importantly, in 6/8 patients, NGS-MRD was detectable between 20 to 220 days (mean 40 days) before clinical relapse (Table 3, Figure 3 and Supplementary Materials Figure S5). 
A

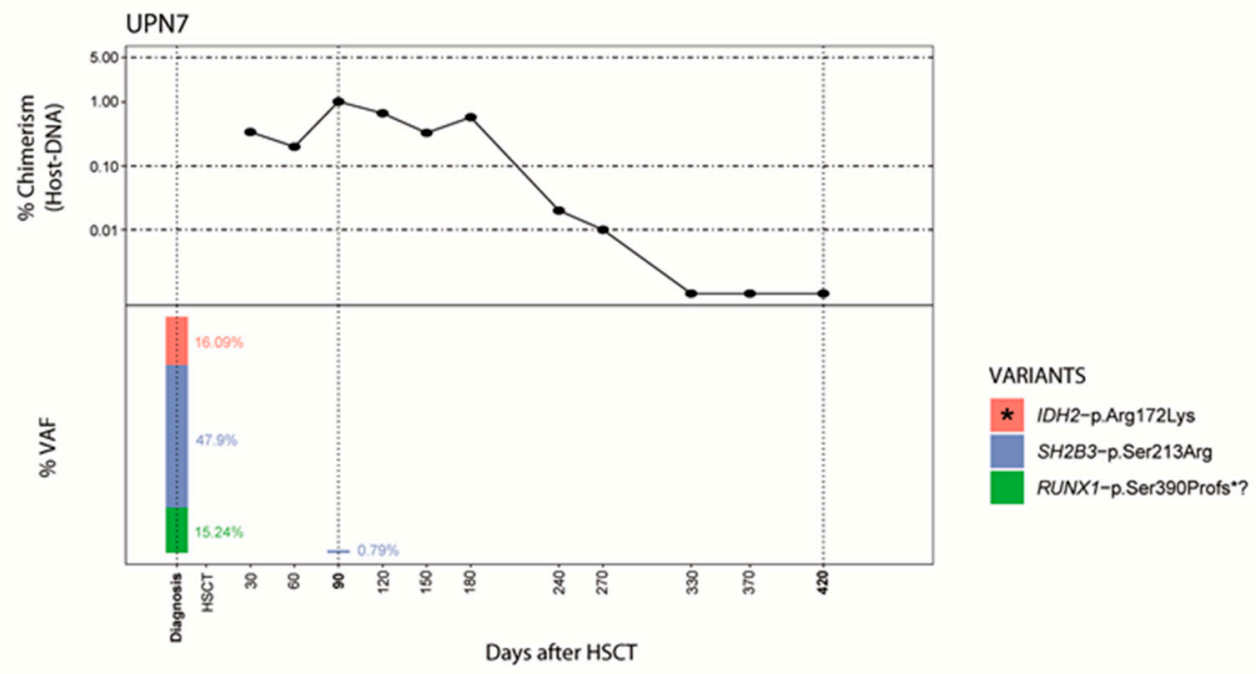

B

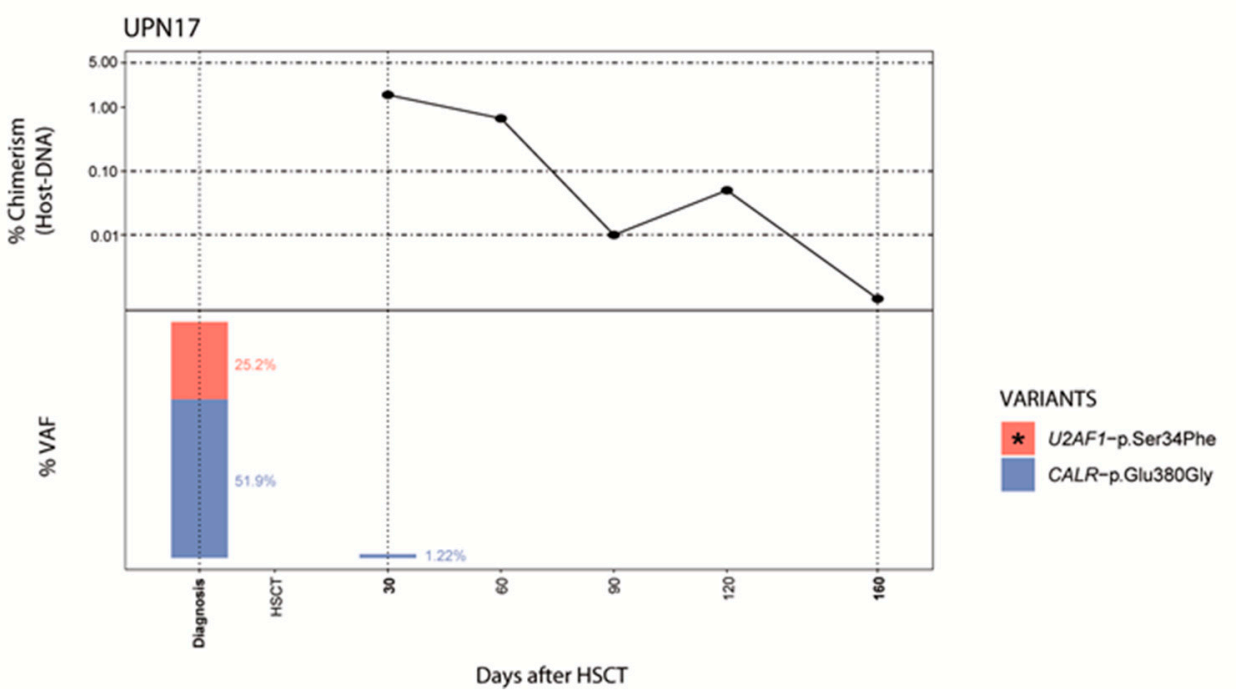

Figure 1. Specificity of the NGS-MRD analysis in non-relapsed patients. Specific negative NGS-MRD confirms remission during MC decreased until CC is reached in both UPN7 (A) and UPN17 (B). Post-HSCT engraftment analysis by indel-qPCR results are plotted as percentage of receptor ( $Y$-axis) over time shown as days post-HSCT (X-axis). Vertical dotted lines denote the NGS-analysis time points and the height bars represents VAF percentages; asterisk indicate NGS-MRD variants. (NGS = next generation sequencing; $\mathrm{MRD}=$ minimal residual disease; $\mathrm{MC}=$ mixed chimerism; $\mathrm{CC}=$ complete chimerism; HSCT = hematopoietic stem cell transplant; UPN = unique patient number; VAF = variant allele frequency).

In 4 relapsed cases, no NGS-MRD was detected: in UPN5 (1.4\% host-DNA) and UPN18 (MC > 5\%), VUS in GATA2 and CUX1 respectively were detected; in UPN4, early relapse was detected with a low MC value (0.2\% host-DNA) and was quickly treated; and in UPN11, NGS-MRD was not detected despite the fact the MC value was high (Supplementary Materials Figure S6).

These results showed that the high specificity of tracking the same NGS variants during HSCT follow up when an increase in $\mathrm{MC}$ is detected could help to discriminate early relapse, providing a useful tool for personalized therapeutic intervention. 
A

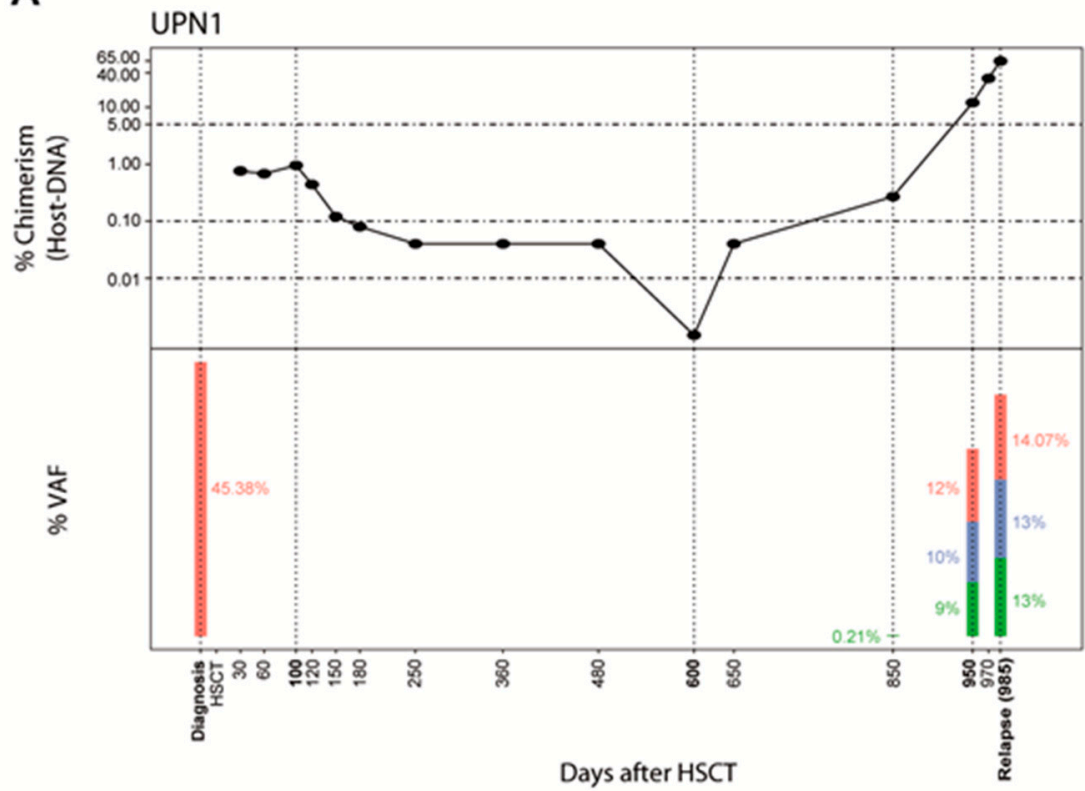

VARIANTS

* NRAS-p.Gin61Lys WT1-p.Arg363Thrfs*5 WT1-p.Ser364Ter

B

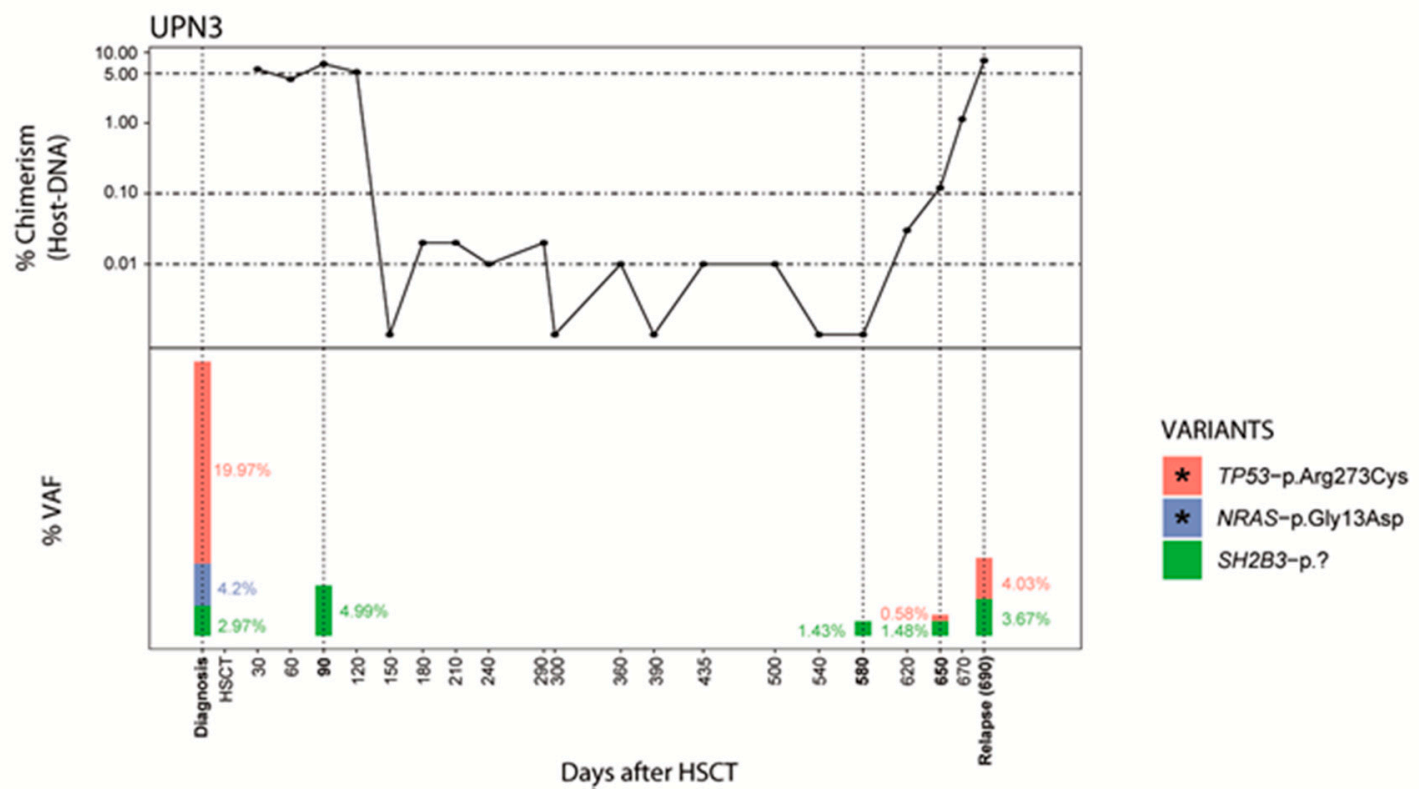

Figure 2. NGS-MRD markers for relapse detection in patients that achieved complete chimerism. Relapsed patients showed a correlation of chimerism status and NGS-MRD during the monitoring of the disease course; MC increase and NGS-MRD variants were detected prior to clinical relapse. (A) In UPN1, negative NGS-MRD correlated with CC and two new variants were detected with the slight increase of MC even before positive NGS-MRD presence. (B) In UPN3, no complete clearance of all the variants was achieved even during CC, and NGS-MRD turned positive when a slight increase of MC was detected. Post-HSCT engraftment analysis by indel-qPCR results are plotted as percentage of receptor ( $Y$-axis) over time shown as days post-HSCT (X-axis). Vertical dotted lines denote the NGS-analysis time points and the height bars represents VAF percentages; asterisk indicate NGS-MRD variants. (NGS = next generation sequencing; $\mathrm{MRD}=$ minimal residual disease; $\mathrm{MC}=$ mixed chimerism; $\mathrm{CC}=$ complete chimerism; $\mathrm{HSCT}$ = hematopoietic stem cell transplant; $\mathrm{UPN}=$ unique patient number; $\mathrm{VAF}=$ variant allele frequency). 
A

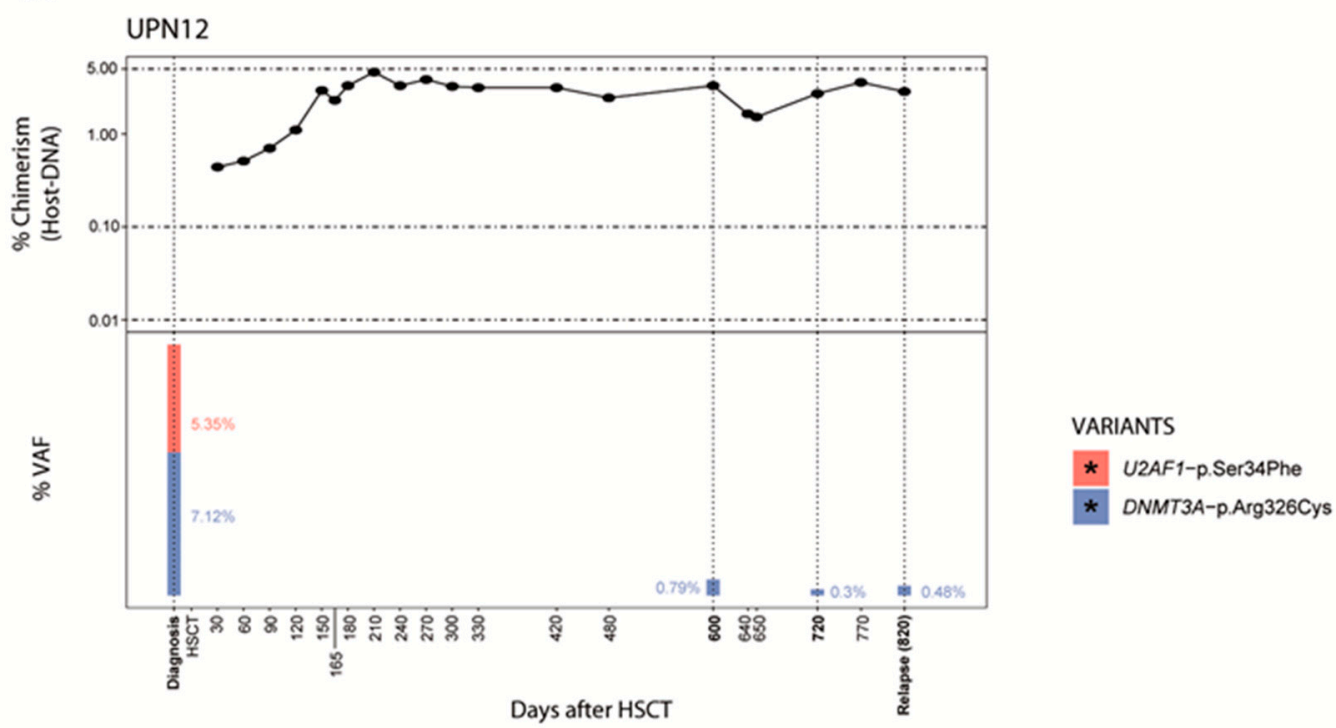

B

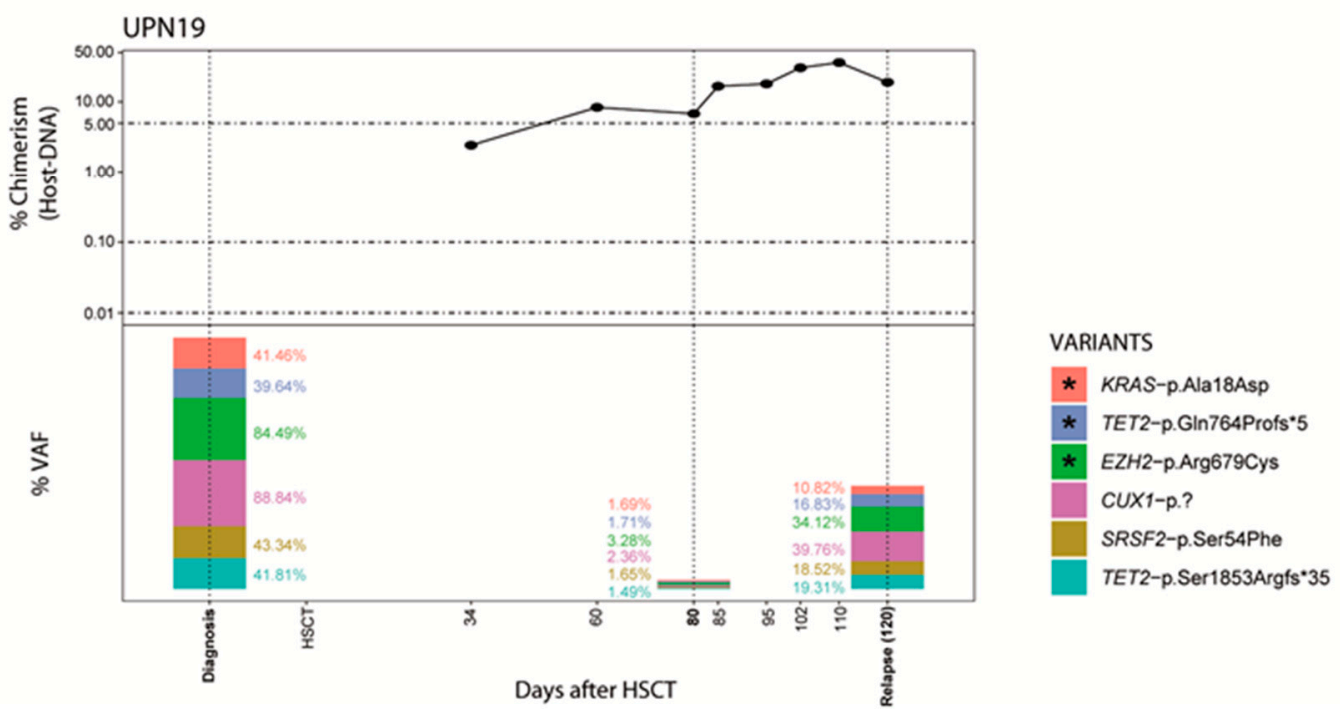

Figure 3. NGS-MRD markers for relapse detection in relapsed patients with MC fluctuations. NGS-MRD during MC monitoring helps to anticipate clinical relapse. Detection of positive NGS-MRD anticipates relapse 220 days in UPN12 (A) and 40 days in UPN19 (B). Post-HSCT engraftment analysis by indel-qPCR results are plotted as percentage of receptor ( $Y$-axis) over time shown as days post-HSCT (X-axis). Vertical dotted lines denote the NGS-analysis time points and the height bars represents VAF percentages; asterisk indicate NGS-MRD variants. (NGS = next generation sequencing; $\mathrm{MRD}=$ minimal residual disease; $\mathrm{MC}=$ mixed chimerism; $\mathrm{CC}=$ complete chimerism; $\mathrm{HSCT}=$ hematopoietic stem cell transplant; UPN = unique patient number; $\mathrm{VAF}=$ variant allele frequency).

\section{Discussion}

The present study aims to investigate the clinical value of post-HSCT NGS-MRD monitoring on serial PB samples in patients with myeloid neoplasms according to chimerism status. Clinical decisions after HSCT, such as lymphocyte donor infusion or removal of immunosuppression, are partially based on chimerism results. Considering that MC can have different clinical implications, including disease relapse, graft failure, and rejection, but may also remain stable for a long time and be compatible with prolonged remission [34], identification of patients who could benefit from an early clinical intervention 
is necessary. We have focused on patients with low levels of MC in hope that close monitoring and NGS-MRD detection could help to take specific clinical decisions such as better timing for the initiation of antineoplastic treatment.

qPCR is as a sensitive method to detect chimerism and previous studies have established cut-off values or increased MC values as a predictive marker for relapse [35-37]. In our cohort, NGS provided useful information to understand clinical status during MC fluctuations and the kinetics of early relapse. Our results suggest that the decision of therapeutic intervention in patients with low levels of MC should be based not only in a defined cut-off value, but also in the individualized chimerism kinetics. For instance, NGS could help to discriminate between MC status with positive NGS-MRD (UPN3) and without positive NGS-MRD (UPN7) (Figures 1 and 2).

Moreover, the use of techniques with higher sensitivity and changes in treatment such as reduced intensity conditioning regimens and T-cell depletion [38] have increased the chances to detect the presence of MC. In our cohort, all patients had MC status after HSCT and the time to achieve CC ranged from $90-600$ days, considering $0.01 \%$ threshold and $70-240$ days with a limit of $0.1 \%$. Therefore, chimerism status needs to be comprehensively interpreted and it is desirable to combine it with an additional method that increases specificity. We have showed the NGS utility in 6 non-relapsed patients where MC was not accompanied with NGS-MRD variants, and in one patient where NGS-MRD variants disappeared when CC was achieved (Figure 1). These findings indicate that the disease course is effectively monitored through combination of both techniques and personalized therapy measures can be implemented if needed.

Different studies showed that the presence of allelic burden by NGS at day 21 post-HSCT can estimate the risk of relapse and mortality, and that NGS-MRD monitoring in PB on days 90 and 180 post-HSCT is predictive for relapse and overall survival $[39,40]$. Our study has demonstrated that monitoring allelic burden by NGS during the disease course is useful to define molecular relapse, and thus could help to take therapeutic decisions. We detected specific positive NGS-MRD in $67 \%$ of the patients with relapse and, importantly, in 6 patients, it was detected between 20 to 220 days before clinical relapse (Table 3).

This finding supports similar results showing positive NGS-MRD in $62 \%$ of 58 samples ( 39 patients) collected 20-80 days prior to relapse [41]. Most NGS panels set their sensitivity around $1 \%$ of VAF for SNV variants, implying that NGS would not be a suitable technology for MRD detection. However, we found that detecting the same NGS variants present at diagnosis during the follow up after HSCT was useful for clinicians to raise a red flag and keep a closer monitorization.

Besides, personalized chimerism monitoring revealed that a slight increase of $\mathrm{MC}(<1 \%)$ detected by the high-sensitive indel-qPCR method, not detectable with STR-PCR (sensitivity $1-5 \%$ ), could identify the accurate timing to perform NGS. Recently, simultaneous variant and single nucleotide polymorphism (SNP) based chimerism NGS study in 14 MDS patients detected an increase of MC and variants in 3 patients with relapse [42]. However, SNP-based chimerism sensitivity is lower than with indel-qPCR, and the cost of several serial samples analysis by NGS will be too high to be implemented in the clinical routine. Similarly, simultaneous molecular and chimerism detection by ddPCR has been demonstrated as a suitable approach for disease monitoring post-HSCT in AML [43]. However, ddPCR limits the number of molecular markers that can be assessed, and new clonal variants indicating progression, like the ones found in UPN1, could be missed.

Importantly, 8 patients had variants in CHIP-associated genes [32] at relapse or the last moment of follow up. Nowadays, these variants are difficult to interpret in the context of the disease progression, so further studies are needed to help to discriminate CHIP variants from clonal disease variants. Besides, it has been previously published that clonal hematopoiesis of donor origin cells may be detected [33]. Altogether, we demonstrate that the evaluation of CHIP variants must be done carefully and that the complete genotyping of donors should be implemented.

Importantly, we have used the same PB DNA samples to analyze chimerism and variant status, showed that they perform similarly to BM DNA, and demonstrated the convenience of combining 
both methods (Table 3). Therefore, the more accessible PB samples could be used to detect MC increase to determine the precise timing to perform NGS, and allow a cost-benefit use of this technique. Overall, we have defined an approach based on NGS-MRD analysis when slight changes of chimerism in PB samples are observed, combining the high-specificity NGS with high-sensitivity chimerism technology.

Despite the advantages of the proposed approach, our patient cohort was limited and therefore we were not able to establish solid values for sensitivity, specificity, and prediction of relapse. Besides, in few relapsed cases, no NGS-MRD was detected, maybe due to the different sensitivity of the technology among variants types (SNV or INDELS) or the fact that some patients may relapse with variants in genes not included in the panel. Therefore, future studies using larger cohorts with serial samples following HSCT would be needed to further confirm the suitability and sensitivity of NGS during chimerism monitoring.

In summary, NGS offers a deeper understanding on variant dynamics throughout the course of post-HSCT and its clinical relevance. Overall, regardless the reason of relapse, the treatment, or the prognosis, this small series shows that personalized NGS-MRD monitoring in combination with highly-sensitive-chimerism analysis are complementary tools to assess early relapse, providing valuable information to monitor myeloid patients after HSCT.

Supplementary Materials: The following are available online at http:/www.mdpi.com/2077-0383/9/12/3818/s1. Figure S1: Flow-chart showing a description of patients and samples selection. Figure S2: Correlation analysis of VAF percentage in peripheral blood and bone marrow-paired samples performed with Pearson correlation test. Figure S3: NGS analysis in non-relapsed patients achieving CC UPN6 (A), UPN10 (B), UPN15 (C), UPN16 (D). Figure S4: NGS analysis in non-relapsed patients with MC. Non-relapsed patients showed negative NGS-MRD despite presence of MC for patients UPN8 (A) and UPN9 (B). Figure S5: Relapsed patients with positive NGS-MRD UPN2 (A), UPN13 (B), UPN14 (C), UPN20 (D). Figure S6: Relapsed patients with no positive NGS-MRD. No NGS-MRD variants were detected at the time of relapse for UPN5 (A), UPN4 (B), UPN11 (C), UPN18 (D).

Author Contributions: Conceptualization, M.F.-M., M.C.V., M.T.Z., I.V., E.B.; methodology, E.B., I.V.; software, B.A.; formal analysis, P.A.-R., Z.B.-I., P.A., A.J.; investigation, P.A.-R., B.A., M.C.V., M.T.Z., Z.B.-I., A.A.-D., A.M., M.J.L., P.A., A.J., M.C.M., M.R.; resources.; M.C.V.; M.T.Z.; M.C.M., M.J.L.; data curation, P.A.-R.; B.A.; writing-original draft preparation, P.A.-R., B.A., E.B., I.V.; writing-review and editing, P.A.-R., M.F.-M., E.B., I.V., F.P.; supervision, M.J.C., F.P., M.C.M., M.R.; project administration, M.F.-M., E.B.; funding acquisition, M.J.C., F.P., M.C.M., M.R. All authors have read and agreed to the published version of the manuscript.

Funding: This work was funded by the Government of Navarra, Department of Industry, Energy and Innovation (Project DIANA, 0011-1411-2017-000028); and supported by CIMA LAB diagnostics research program. F.P. acknowledges funding from Instituto de Salud Carlos III (ISCIII) PI16/02024, PI17/00701 and PI19/01352 (Co-financed with European Union FEDER funds), CIBERONC CB16/12/00489 (Co-financed with European Union FEDER funds), MINECO Explora (RTHALMY), Departamento de Salud-Gobierno de Navarra 40/2016 and Fundación Ramón Areces (PREMAMM).

Acknowledgments: We acknowledge the support given by the CIMA LAB Diagnostics team members. We particularly acknowledge the patients for their participation.

Conflicts of Interest: The authors declare no conflict of interest. The funders had no role in the design of the study; in the collection, analyses, or interpretation of data; in the writing of the manuscript, or in the decision to publish the results.

\section{References}

1. Cornelissen, J.J.; Gratwohl, A.; Schlenk, R.F.; Sierra, J.; Bornhäuser, M.; Juliusson, G.; Råcil, Z.; Rowe, J.M.; Russell, N.; Mohty, M.; et al. The European LeukemiaNet AML Working Party consensus statement on allogeneic HSCT for patients with AML in remission: An integrated-risk adapted approach. Nat. Rev. Clin. Oncol. 2012, 9, 579-590. [CrossRef] [PubMed]

2. Schlenk, R.F.; Döhner, K.; Mack, S.; Stoppel, M.; Király, F.; Götze, K.; Hartmann, F.; Horst, H.A.; Koller, E.; Petzer, A.; et al. Prospective Evaluation of Allogeneic Hematopoietic Stem-Cell Transplantation from Matched Related and Matched Unrelated Donors in Younger Adults with High-Risk Acute Myeloid Leukemia: German-Austrian Trial AMLHD98A. J. Clin. Oncol. 2010, 28, 4642-4648. [CrossRef] 
3. Cornelissen, J.J.; Van Putten, W.L.J.; Verdonck, L.F.; Theobald, M.; Jacky, E.; Daenen, S.M.G.; Kooy, M.V.M.; Wijermans, P.; Schouten, H.; Huijgens, P.C.; et al. Results of a HOVON/SAKK donor versus no-donor analysis of myeloablative HLA-identical sibling stem cell transplantation in first remission acute myeloid leukemia in young and middle-aged adults: Benefits for whom? Blood 2007, 109, 3658-3666. [CrossRef] [PubMed]

4. Scott, B.L.; Pasquini, M.C.; Logan, B.R.; Wu, J.; Devine, S.M.; Porter, D.L.; Maziarz, R.T.; Warlick, E.D.; Fernandez, H.F.; Alyea, E.P.; et al. Myeloablative Versus Reduced-Intensity Hematopoietic Cell Transplantation for Acute Myeloid Leukemia and Myelodysplastic Syndromes. J. Clin. Oncol. 2017, 35, 1154-1161. [CrossRef] [PubMed]

5. Buccisano, F.; Maurillo, L.; Del Principe, M.I.; Del Poeta, G.; Sconocchia, G.; Lo-Coco, F.; Arcese, W.; Amadori, S.; Venditti, A. Prognostic and therapeutic implications of minimal residual disease detection in acute myeloid leukemia. Blood 2012, 119, 332-341. [CrossRef]

6. Schlenk, R.F.; Kayser, S.; Bullinger, L.; Kobbe, G.; Casper, J.; Ringhoffer, M.; Held, G.; Brossart, P.; Lübbert, M.; Salih, H.R.; et al. Differential impact of allelic ratio and insertion site in FLT3-ITD-positive AML with respect to allogeneic transplantation. Blood 2014, 124, 3441-3449. [CrossRef]

7. Kongtim, P.; Hasan, O.; Perez, J.M.R.; Varma, A.; Wang, S.A.; Patel, K.P.; Chen, J.; Rondon, G.; Srour, S.; Bashir, Q.; et al. Novel Disease Risk Model for Patients with Acute Myeloid Leukemia Receiving Allogeneic Hematopoietic Cell Transplantation. Biol. Blood Marrow Transplant. 2020, 26, 197-203. [CrossRef]

8. Araki, D.; Wood, B.L.; Othus, M.; Radich, J.P.; Halpern, A.B.; Zhou, Y.; Mielcarek, M.; Estey, E.H.; Appelbaum, F.R.; Walter, R.B. Allogeneic Hematopoietic Cell Transplantation for Acute Myeloid Leukemia: Time to Move Toward a Minimal Residual Disease-Based Definition of Complete Remission? J. Clin. Oncol. 2016, 34, 329-336. [CrossRef]

9. Choi, S.-J.; Lee, K.-H.; Lee, J.-H.; Kim, S.-H.; Chung, H.-J.; Park, C.-J.; Chi, H.-S.; Kim, W.-K. Prognostic value of hematopoietic chimerism in patients with acute leukemia after allogeneic bone marrow transplantation: A prospective study. Bone Marrow Transplant. 2000, 26, 327-332. [CrossRef]

10. Mosna, F.; Capelli, D.; Gottardi, M. Minimal Residual Disease in Acute Myeloid Leukemia: Still a Work in Progress? J. Clin. Med. 2017, 6, 57. [CrossRef]

11. Schuurhuis, G.J.; Heuser, M.; Freeman, S.; Béné, M.-C.; Lo-Coco, F.; Cloos, J.; Grimwade, D.; Haferlach, T.; Hills, R.K.; Hourigan, C.S.; et al. Minimal/measurable residual disease in AML: A consensus document from the European LeukemiaNet MRD Working Party. Blood 2018, 131, 1275-1291. [CrossRef] [PubMed]

12. Fernández-Avilés, F.; Urbano-Ispizua, A.; Aymerich, M.; Colomer, D.; Rovira, M.; Martinez, C.; Nadal, E.; Talarn, C.; Carreras, E.; Montserrat, E. Serial quantification of lymphoid and myeloid mixed chimerism using multiplex PCR amplification of short tandem repeat-markers predicts graft rejection and relapse, respectively, after allogeneic transplantation of CD34+ selected cells from peripheral blood. Leukemia 2003, 17, 613-620. [CrossRef] [PubMed]

13. Sufliarska, S.; Minarik, G.; Horakova, J.; Bodova, I.; Bojtarova, E.; Czako, B.; Mistrik, M.; Drgona, L.; Demitrovicova, M.; Lakota, J.; et al. Establishing the method of chimerism monitoring after allogeneic stem cell transplantation using multiplex polymerase chain reaction amplification of short tandem repeat markers and Amelogenin. Neoplasma 2007, 54, 424-430. [PubMed]

14. Thiede, C.; Bornhauser, M.; Ehninger, G. Evaluation of STR informativity for chimerism testing-comparative analysis of 27 STR systems in 203 matched related donor recipient pairs. Leukepia 2004, 18, 248-254. [CrossRef]

15. Alizadeh, M.; Bernard, M.; Danic, B.; Dauriac, C.; Birebent, B.; Lapart, C.; Lamy, T.; Le Prisé, P.-Y.; Beauplet, A.; Bories, D.; et al. Quantitative assessment of hematopoietic chimerism after bone marrow transplantation by real-time quantitative polymerase chain reaction. Blood 2002, 99, 4618-4625. [CrossRef]

16. Jiménez-Velasco, A.; Román-Gómez, J.; Agirre, X.; Barrios, M.; Navarro, G.; Vázquez, I.; Prósper, F.; Torres, A.; Heiniger, A. Downregulation of the large tumor suppressor 2 (LATS2/KPM) gene is associated with poor prognosis in acute lymphoblastic leukemia. Leukepia 2005, 19, 2347-2350. [CrossRef]

17. Kim, S.Y.; Jeong, M.H.; Park, N.; Ra, E.; Park, H.; Seo, S.H.; Kim, J.Y.; Seong, M.-W.; Park, S.S. Chimerism Monitoring after Allogeneic Hematopoietic Stem Cell Transplantation Using Quantitative Real-Time PCR of Biallelic Insertion/Deletion Polymorphisms. J. Mol. Diagn. 2014, 16, 679-688. [CrossRef]

18. Shimoni, A.; Nagler, A.; Kaplinsky, C.; Reichart, M.; Avigdor, A.; Hardan, I.; Yeshurun, M.; Daniely, M.; Zilberstein, Y.; Amariglio, N.; et al. Chimerism testing and detection of minimal residual disease after allogeneic hematopoietic transplantation using the bioView (Duet ${ }^{\mathrm{TM}}$ ) combined morphological and cytogenetical analysis. Leukepia 2002, 16, 1413-1418. [CrossRef] 
19. Jacobsohn, D.A.; Loken, M.R.; Fei, M.; Adams, A.; Brodersen, L.E.; Logan, B.R.; Ahn, K.W.; Shaw, B.E.; Kletzel, M.; Olszewski, M.; et al. Outcomes of Measurable Residual Disease in Pediatric Acute Myeloid Leukemia before and after Hematopoietic Stem Cell Transplant: Validation of Difference from Normal Flow Cytometry with Chimerism Studies and Wilms Tumor 1 Gene Expression. Biol. Blood Marrow Transplant. 2018, 24, 2040-2046. [CrossRef]

20. Papaemmanuil, E.; Gerstung, M.; Bullinger, L.; Gaidzik, V.I.; Paschka, P.; Roberts, N.D.; Potter, N.E.; Heuser, M.; Thol, F.; Bolli, N.; et al. Genomic Classification and Prognosis in Acute Myeloid Leukemia. N. Engl. J. Med. 2016, 374, 2209-2221. [CrossRef]

21. Jongen-Lavrencic, M.; Grob, T.; Hanekamp, D.; Kavelaars, F.G.; Al Hinai, A.; Zeilemaker, A.; Erpelinck-Verschueren, C.A.; Gradowska, P.L.; Meijer, R.; Cloos, J.; et al. Molecular Minimal Residual Disease in Acute Myeloid Leukemia. N. Engl. J. Med. 2018, 378, 1189-1199. [CrossRef] [PubMed]

22. Getta, B.M.; Devlin, S.M.; Levine, R.L.; Arcila, M.E.; Mohanty, A.S.; Zehir, A.; Tallman, M.S.; Giralt, S.A.; Roshal, M. Multicolor Flow Cytometry and Multigene Next-Generation Sequencing Are Complementary and Highly Predictive for Relapse in Acute Myeloid Leukemia after Allogeneic Transplantation. Biol. Blood Marrow Transplant. 2017, 23, 1064-1071. [CrossRef] [PubMed]

23. Press, R.D.; Eickelberg, G.; Froman, A.; Yang, F.; Stentz, A.; Flatley, E.M.; Fan, G.; Lim, J.Y.; Meyers, G.; Maziarz, R.T.; et al. Next-generation sequencing-defined minimal residual disease before stem cell transplantation predicts acute myeloid leukemia relapse. Am. J. Hematol. 2019, 94, 902-912. [CrossRef] [PubMed]

24. Spencer, D.H.; Ketkar-kulkarni, S.; Wartman, L.D.; Christopher, M.; Lamprecht, T.L.; Helton, N.M.; Eric, J.; Payton, J.E.; Baty, J.; Heath, S.E.; et al. Association Between Mutation Clearance After Induction Therapy and Outcomes in Acute Myeloid Leukemia. JAMA 2016, 314, 811-822. [CrossRef]

25. Morita, K.; Kantarjian, H.M.; Wang, F.; Yan, Y.; Bueso-Ramos, C.; Sasaki, K.; Issa, G.C.; Wang, S.; Jorgensen, J.; Song, X.; et al. Clearance of Somatic Mutations at Remission and the Risk of Relapse in Acute Myeloid Leukemia. J. Clin. Oncol. 2018, 36, 1788-1797. [CrossRef] [PubMed]

26. Yoshizato, T.; Nannya, Y.; Atsuta, Y.; Shiozawa, Y.; Iijima-Yamashita, Y.; Yoshida, K.; Shiraishi, Y.; Suzuki, H.; Nagata, Y.; Sato, Y.; et al. Genetic abnormalities in myelodysplasia and secondary acute myeloid leukemia: Impact on outcome of stem cell transplantation. Blood 2017, 129, 2347-2358. [CrossRef]

27. Gendzekhadze, K.; Gaidulis, L.; Senitzer, D. Chimerism Testing by Quantitative PCR Using Indel Markers. In Transplantation Immunology; Humana Press: Totowa, NJ, USA, 2013; Volume 1034, pp. 221-237. [CrossRef]

28. Aguilera-Diaz, A.; Vazquez, I.; Ariceta, B.; Mañú, A.; Blasco-Iturri, Z.; Palomino-Echeverría, S.; Larrayoz, M.J.; García-Sanz, R.; Prieto-Conde, M.I.; Chillón, M.D.C.; et al. Assessment of the clinical utility of four NGS panels in myeloid malignancies. Suggestions for NGS panel choice or design. PLoS ONE 2020, 15, e0227986. [CrossRef]

29. Koboldt, D.C.; Zhang, Q.; Larson, D.E.; Shen, D.; McLellan, M.D.; Lin, L.; Miller, C.A.; Mardis, E.R.; Ding, L.; Wilson, R.K. VarScan 2: Somatic mutation and copy number alteration discovery in cancer by exome sequencing. Genome Res. 2012, 22, 568-576. [CrossRef]

30. Cibulskis, K.; Lawrence, M.S.; Carter, S.L.; Sivachenko, A.; Jaffe, D.B.; Sougnez, C.; Gabriel, S.B.; Meyerson, M.L.; Lander, E.S.; Getz, G. Sensitive detection of somatic point mutations in impure and heterogeneous cancer samples. Nat. Biotechnol. 2013, 31, 213-219. [CrossRef]

31. Palomo, L.; Ibáñez, M.; Abáigar, M.; Vázquez, I.; Álvarez, S.; Cabezón, M.; Tazón-Vega, B.; Rapado, I.; Fuster-Tormo, F.; Cervera, J.; et al. Spanish Guidelines for the use of targeted deep sequencing in myelodysplastic syndromes and chronic myelomonocytic leukaemia. Br. J. Haematol. 2019, 188, 605-622. [CrossRef]

32. Steensma, D.P.; Bejar, R.; Jaiswal, S.; Lindsley, R.C.; Sekeres, M.A.; Hasserjian, R.P.; Ebert, B.L. Clonal hematopoiesis of indeterminate potential and its distinction from myelodysplastic syndromes. Blood 2015, 126, 9-16. [CrossRef] [PubMed]

33. Hasserjian, R.P.; Steensma, D.P.; Graubert, T.A.; Ebert, B.L. Clonal hematopoiesis and measurable residual disease assessment in acute myeloid leukemia. Blood 2020, 135, 1729-1738. [CrossRef] [PubMed]

34. Schaap, N.P.M.; Schattenberg, A.; Mensink, E.; Preijers, F.; Hillegers, M.; Knops, R.; Pennings, A.; Boezeman, J.; Van Kessel, A.G.; De Pauw, B.; et al. Long-term follow-up of persisting mixed chimerism after partially T cell-depleted allogeneic stem cell transplantation. Leukepia 2002, 16, 13-21. [CrossRef] [PubMed] 
35. Ahci, M.; Stempelmann, K.; Buttkereit, U.; Crivello, P.; Trilling, M.; Heinold, A.; Steckel, N.K.; Koldehoff, M.; Horn, P.A.; Beelen, D.W.; et al. Clinical Utility of Quantitative PCR for Chimerism and Engraftment Monitoring after Allogeneic Stem Cell Transplantation for Hematologic Malignancies. Biol. Blood Marrow Transplant. 2017, 23, 1658-1668. [CrossRef]

36. Sellmann, L.; Rabe, K.; Bünting, I.; Dammann, E.; Göhring, G.; Ganser, A.; Stadler, M.; Weissinger, E.M.; Hambach, L. Diagnostic value of highly-sensitive chimerism analysis after allogeneic stem cell transplantation. Bone Marrow Transplant. 2018, 53, 1457-1465. [CrossRef]

37. Jacque, N.; Nguyen, S.; Golmard, J.-L.; Uzunov, M.; Garnier, A.; Leblond, V.; Vernant, J.-P.; Bories, D.; Dhédin, N. Chimerism analysis in peripheral blood using indel quantitative real-time PCR is a useful tool to predict post-transplant relapse in acute leukemia. Bone Marrow Transplant. 2014, 50, 259-265. [CrossRef]

38. Bouvier, A.; Ribourtout, B.; François, S.; Orvain, C.; Paz, D.L.; Beucher, A.; Guérard, A.; Guardiola, P.; Ugo, V.; Blanchet, O.; et al. Donor cell-derived acute promyelocytic leukemia after allogeneic hematopoietic stem cell transplantation. Eur. J. Haematol. 2018, 101, 570-574. [CrossRef]

39. Kim, T.; Moon, J.H.; Ahn, J.-S.; Kim, Y.-K.; Lee, S.-S.; Ahn, S.-Y.; Jung, S.-H.; Yang, D.-H.; Lee, J.-J.; Choi, S.H.; et al. Next-generation sequencing-based posttransplant monitoring of acute myeloid leukemia identifies patients at high risk of relapse. Blood 2018, 132, 1604-1613. [CrossRef] [PubMed]

40. Thol, F.; Gabdoulline, R.; Liebich, A.; Klement, P.; Schiller, J.; Kandziora, C.; Hambach, L.; Stadler, M.; Koenecke, C.; Flintrop, M.; et al. Measurable residual disease monitoring by NGS before allogeneic hematopoietic cell transplantation in AML. Blood 2018, 132, 1703-1713. [CrossRef] [PubMed]

41. Balagopal, V.; Hantel, A.; Kadri, S.; Steinhardt, G.; Zhen, C.J.; Kang, W.; Wanjari, P.; Ritterhouse, L.L.; Stock, W.; Segal, J.P. Measurable residual disease monitoring for patients with acute myeloid leukemia following hematopoietic cell transplantation using error corrected hybrid capture next generation sequencing. PLoS ONE 2019, 14, e0224097. [CrossRef] [PubMed]

42. Lee, J.-M.; Kim, Y.-J.; Park, S.-S.; Han, E.; Kim, M.; Kim, Y. Simultaneous Monitoring of Mutation and Chimerism Using Next-Generation Sequencing in Myelodysplastic Syndrome. J. Clin. Med. 2019, 8, 2077. [CrossRef] [PubMed]

43. Waterhouse, M.; Pfeifer, D.; Duque-Afonso, J.; Follo, M.; Duyster, J.; Depner, M.; Bertz, H.; Finke, J. Droplet digital PCR for the simultaneous analysis of minimal residual disease and hematopoietic chimerism after allogeneic cell transplantation. Clin. Chem. Lab. Med. 2019, 57, 641-647. [CrossRef] [PubMed]

Publisher's Note: MDPI stays neutral with regard to jurisdictional claims in published maps and institutional affiliations.

(C) 2020 by the authors. Licensee MDPI, Basel, Switzerland. This article is an open access article distributed under the terms and conditions of the Creative Commons Attribution (CC BY) license (http://creativecommons.org/licenses/by/4.0/). 